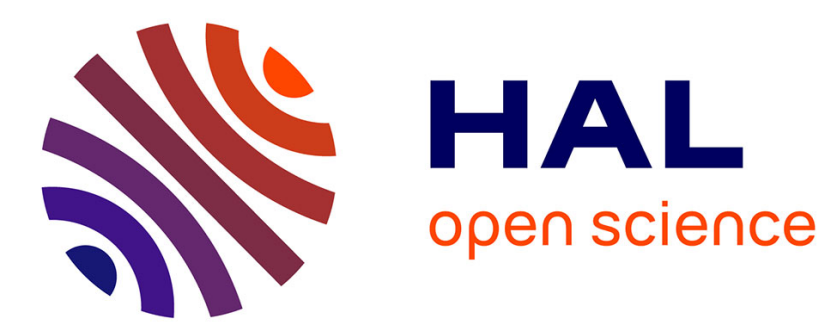

\title{
An integrated framework for operational flexibility assessment in multi-period power system planning with renewable energy production
}

Islam Abdin, Enrico Zio

\section{- To cite this version:}

Islam Abdin, Enrico Zio. An integrated framework for operational flexibility assessment in multiperiod power system planning with renewable energy production. Applied Energy, 2018, 222, pp.898914. 10.1016/j.apenergy.2018.04.009 . hal-01988983

\section{HAL Id: hal-01988983 \\ https://hal.science/hal-01988983}

Submitted on 8 Feb 2019

HAL is a multi-disciplinary open access archive for the deposit and dissemination of scientific research documents, whether they are published or not. The documents may come from teaching and research institutions in France or abroad, or from public or private research centers.
L'archive ouverte pluridisciplinaire $\mathbf{H A L}$, est destinée au dépôt et à la diffusion de documents scientifiques de niveau recherche, publiés ou non, émanant des établissements d'enseignement et de recherche français ou étrangers, des laboratoires publics ou privés. 


\title{
An integrated framework for operational flexibility assessment in multi- period power system planning with renewable energy production
}

\author{
Islam F. Abdin ${ }^{\mathrm{a}}$, Enrico Zio ${ }^{\mathrm{a}, \mathrm{b}, *}$ \\ ${ }^{a}$ Laboratoire Genie Industriel, CentraleSupélec, Université Paris-Saclay, 3 Rue Joliot Curie, 91190 Gif-sur-Yvette, France, Chair on Systems Science and the Energy \\ Challenge, Fondation Electricité de France (EDF), France \\ ${ }^{\mathrm{c}}$ Department of Energy, Politecnico di Milano, Italy
}

\section{H I G H L I G H T S}

- Quantitative modeling framework for operational flexibility assessment.

- Integration of short-term constraints in generation expansion planning model.

- Renewable energy penetration drives flexibility needs higher than carbon limits.

\section{A R T I C L E I N F O}

\section{Keywords:}

Operational flexibility

Flexibility metrics

Generation expansion planning

Unit commitment

Renewable energy penetration

Optimization

\begin{abstract}
A B S T R A C T
This paper proposes an integrated framework for operational flexibility assessment in power system planning with a significant share of intermittent renewable energy sources (RES). The framework proposed includes: (i) the formulation of an integrated generation expansion planning (GEP) and unit commitment (UC) model accounting for key short-term technical constraints, (ii) the elaboration of accurate and systematic horizon reduction methods to alleviate the computational burden of the resulting large-sized optimization problems and (iii) the definition of suitable metrics for the operational flexibility assessment of the obtained plans. The framework is applied to a ten year planning horizon of a realistically sized case study representing the national power system of France, under several scenarios of RES penetration levels and carbon limits, spanning levels of up to $50 \%$. The importance of incorporating the detailed short-term constraints within long-term planning models is shown. The results of the assessment show that, under high renewable energy penetration, neglecting the short-term constraints may lead to plans significantly short on flexibility, reaching shortage levels of up to $50 \%$ in frequency and several GWs in magnitude. Also, the load not served reaches levels of up to $3 \%$ and carbon emission is underestimated by up to $60 \%$. Furthermore, the results highlight the importance of relying on suitable quantitative metrics for operational flexibility assessment in power systems planning rather than solely relying on generic performance measures, such as system costs and mixes of power plants, which are shown not to sufficiently reflect the flexibility levels of the obtained plans.
\end{abstract}

\section{Introduction}

Generation expansion planning (GEP) is a well studied techno-economic problem, which relates to determining the optimal of generation technologies mix, their siting and their investment schedules, for ensuring that the electricity demand over a certain time horizon can be satisfied. With the power sector being constantly subjected to changes, driven by economical, technical, social and environmental issues, GEP modeling techniques have continuously evolved to accommodate the newly arising requirements. Such modeling advancements have been covered in recent literature reviews and include, among others $[1,2]$ : improvements in the details considered (e.g. reliability and maintenance), policy developments, such as the restructuring of the power sector, renewable energy sources (RES) integration and support schemes, uncertainty and stochasticity modeling, and the consideration of real-options for adaptive power systems design [3].

One of the most recent concerns in power systems planning is dealing with the high share of intermittent RES penetration required in the system, driven by strict environmental policies, such as the EU renewable energy directive [4] and its proposed revision [5], and other regional and national targets. The resulting increased variability in the net load (system demand minus RES production) requires that the

\footnotetext{
* Corresponding author at: Department of Energy, Politecnico di Milano, Italy.

E-mail addresses: islam.abdin@centralesupelec.fr (I.F. Abdin), enrico.zio@centralesupelec.fr, enrico.zio@polimi.it (E. Zio).
} 


\section{List of symbols \\ Indexes \\ $i \quad$ index of power plant cluster \\ $j \quad$ index of sub-periods (hours) \\ $s \quad$ index of sub-periods (load-levels) \\ $y \quad$ index of planning year \\ Sets \\ I set of power plant clusters \\ $I^{\text {new }} \quad$ subset of new power plants cluster \\ $I^{\text {res }} \quad$ subset of renewable energy units cluster \\ $I^{\text {th }} \quad$ subset of thermal and nuclear units cluster \\ $J \quad$ set of hourly sub-periods \\ $S \quad$ set of load-levels sub-periods \\ $Y \quad$ set of years in the planning horizon \\ $\Theta \quad$ set of investment decision variables \\ $\Omega \quad$ set of operation decision variables}

\section{Parameters}

$Y^{\text {end }} \quad$ end year of the planning horizon

$Y^{\text {res }} \quad$ first year during which the RES quota target is binding

$L_{y, *} \quad$ demand at sub-period $j$ or $s$ in year $y$ (MW)

Dury,s duration of load block $s$ in year $y$ (hours)

$P_{i}^{\max } \quad$ maximum capacity of power plant $i$ (MW)

$P_{i}^{\min } \quad$ minimum stable power output of power plant $i \in I^{\text {th }}$ (MW/ h)

$C_{i}^{\text {inv }} \quad$ investment cost of unit $i(\mathrm{M} €)$

$I_{i}^{\max }$ maximum allowable units to be commissioned within the planning horizon

$T_{i}^{\text {life }} \quad$ expected life-time of new power plant $i$ (years)

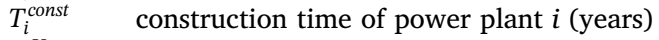

$R_{i}^{\text {Umax }}$ maximum upwards ramping capability of power plant $i \in I^{\text {th }}(\mathrm{MW} / \mathrm{h})$

$R_{i}^{\text {Dmax }}$ maximum downwards ramping capability of power plant $i \in I^{\text {th }}(\mathrm{MW} / \mathrm{h})$

$P_{i}^{\text {start }} \quad$ maximum output of power plant $i \in I^{\text {th }}$ when started (MW)

$C F_{i, y, *} \quad$ capacity factor of renewable energy sources $i \in I^{\text {res }}$ during sub-period $j$ or $s$, of year $y(\%)$

$E_{i} \quad$ amount of carbon emission per MWh of power plant $i$ $\left(t \mathrm{CO}_{2} / \mathrm{MWh}\right)$

$E_{y}^{\max } \quad$ maximum total allowable emission per year y $\left(t C \mathrm{O}_{2}\right)$

$E F O R_{i} \quad$ Expected forced outage rate of power plant $i(\%)$

$M_{i}^{u} \quad$ minimum up-time for power plant $i \in I^{\text {th }}$ (hours)

$M_{i}^{d} \quad$ minimum down-time of power plant $i \in I^{\text {th }}$ (hours)

$D R_{y} \quad$ discount rate for year y (\%)

$C_{i, y}^{\text {mrgl }}$ marginal cost of power plant $i$ including the variable O\&M and $\mathrm{CO}_{2}$ costs, considering inflation ( $\left.€ / \mathrm{MWh}\right)$

$C_{i}^{S} \quad$ start-up cost of power plant $i(€)$

$C^{\text {lns }} \quad$ cost of load not served $(€ / \mathrm{MWh})$

$C_{i}^{\text {fom }} \quad$ fixed O\&M costs of power plant $i(€)$

$P e n_{y}^{\text {level }}$ annual renewable penetration level requirement (\%)

$\mathrm{Prr} \quad$ percentage of the load required to be covered by primary reserve $(\%)$

$S r r^{u p} \quad$ percentage of the load required to be covered by the secondary upwards reserve (\%)

$S r r^{d n} \quad$ percentage of the load required to be covered by the secondary downwards reserve (\%)

$a^{\text {res }} \quad$ percentage of the variable generation output covered by secondary reserve (\%)

$r^{\text {min }} \quad$ minimum planning reserve margin (MW)

Continuous variables

$p_{i, y, *} \quad$ energy output of power plant $i$ at sub-period $j$ or $s$, during year $y$ (MWh)

$p r_{i, y, j} \quad$ primary reserve of unit $i$ at sub-period $j$ during year $y$ (MWh)

$s r_{i, y, j}^{u p} \quad$ secondary upwards reserve of unit $i$ at sub-period $j$ during year $y$ (MWh)

$s r_{i, y, j}^{d n} \quad$ secondary downwards reserve of unit $i$ at sub-period $j$ during year $y$ (MWh)

$\ln s_{y, *} \quad$ load not served at sub-period $j$ or $s$, during year $y$ (MW)

$v_{i, y, j} \quad$ shut-down decision of unit $i$ during sub-period $j$ in year $y$

\section{Discrete variables}

$x_{i, y} \quad$ availability (commissioning) state of power plant $i$ in year

$$
y
$$

$q_{i, y} \quad$ commissioning decision of power plant $i$ in year $y$

$u_{i, y, j} \quad$ commitment status of power plant $i$ during sub-period $j$ in year $y$

$z_{i, y, j} \quad$ start-up decision of power plant $i$ during sub-period $j$ in year $y$

Acronyms

$\begin{array}{ll}\text { CF } & \text { Capacity Factor } \\ \text { EFS } & \text { Expected Flexibility Shortfall } \\ \text { GEP } & \text { Generation Expansion Planning } \\ \text { IRRE } & \text { Insufficient Ramping Resources Expectation } \\ \text { LDC } & \text { Load Duration Curve } \\ \text { LNS } & \text { Load Not Served } \\ \text { MILP } & \text { Mixed Integer Linear Programming } \\ \text { O\&M } & \text { Operation and Maintenance } \\ \text { RES } & \text { Renewable Energy Sources } \\ \text { UC } & \text { Unit Commitment }\end{array}$

remainder of the thermal units cope with tighter operational flexibility requirements $[6,7]$. This is generally defined as the ability of the system to respond to the inter-temporal variability rising both from intermittent RES production and from variations in electricity demand. In this respect, operational flexibility regards the short-term operation of those generation units and their technical characteristics: ramping rates, unit commitment states, minimum up and down times, start-up times and minimum stable load, to name a few.

From an assessment point of view, accounting for operational flexibility is a critical element for overall system reliability (see for example Fulli et al. [8] for a discussion on these requirements in Europe).
Reliability relates to firm-capacity ${ }^{1}$ at each time period sufficient to satisfy the system load, using typical metrics such as loss of load expectation (LOLE) and expected energy not supplied (EENS). Operational flexibility, instead, considers how a specific operational state of the system at a given period would contribute to (or hinder) its ability to deploy its resources for accommodating subsequent load variations. For this, no time period can be assessed in isolation of the others, nor without detailed knowledge of the system state and technical characteristics at the given period.

\footnotetext{
${ }^{1}$ Available generation capacity excluding failed units, units in maintenance, offline units, etc.
} 
Therefore, dedicated metrics to describe operational flexibility have been recently proposed in the literature, varying in the degree of complexity and in the data required for their estimation. Lannoye et al. [9] proposes a probabilistic metric that takes into account key technical characteristics and operational states of the generation units, and aggregates them for a system-level assessment. Oree and Hassen [10] proposes a composite metric which aggregates a set of the generation units flexibility parameters through normalization, weighting and correlation analysis. In [11] a number of interdependent metrics are defined for individual generation units to assess their available flexibility in real time, while [12] proposes a metric which additionally considers the impacts of the transmission network on the flexibility levels.

There is no consensus on which metrics are best adapted to evaluate operational flexibility within the different contexts of power system evaluation. The flexibility challenges caused by RES integration require a delicate balance between the level of details considered and the simplicity of understanding the measurements obtained. Extensive metrics such as those proposed in Ulbig and Andersson [11] and Zhao et al. [12] may be suitable for real-time operational assessment, but would be too laborious to consider within a long-term planning context. Simpler measures that consider key technical details but neglect the operational state of the system may provide useful long-term insights but are limited in accounting for time-specific attributes of flexibility, such as those imposed by RES intermittency. There is, then, a need for developing intermediate level probabilistic metrics, such as that proposed in [9] and used in this work, which itself is limited by considering only the frequency of flexibility shortage while neglecting its magnitude. This is discussed in details in Section 2.4.

With respect to power systems planning, traditional long-term GEP models do not consider the chronological representation of net load variations, nor the short-term technical constraints of the generation units, but rather rely on average system representations. The generation plans obtained are, therefore, not explicitly driven by the requirement to deal with short-term variations. This type of evaluation is, on the contrary, typically performed by the well known unit commitment (UC) problem, which does not consider investment decisions [13]. Accordingly, to account for the operational flexibility aspect, recent planning studies have started to investigate the importance of integrating the short-term constraints within long-term planning models. Belderbos and Delarue [14] solve a traditional GEP model based on a basic screening curve method and propose a perturbation algorithm with embedded short-term constraints to improve the plans obtained. They show that considering the short-term constraints results in the installation of more mid and peak load capacities than the case of neglecting them. Similar results are reported in [15], who solve a combined GEP-UC model for a single future year, under several wind penetration and carbon pricing scenarios. Additionally they show that neglecting short-term constraints leads to an underestimation of carbon emission and wind curtailment levels of the obtained plans. The same general trends are shown in $[16,17]$ for case studies on Ireland and Poland, respectively. For multi-annual planning studies, Koltsaklis and Georgiadis [18] propose a combined model and use it for the future planning of the Greek power system, while Guo et al. [19] propose a similar investigation applied to the Chinese power system. In both cases the results revealed the correlation between significant RES penetration with increased amounts of peaking units investments. Finally, Pereira et al. [20] compare an integrated model to a classical one for a ten year planning period and show that neglecting these constraints underestimates both the investment costs and the emissions levels.

The above studies have investigated the importance of including the short-term constraints within long-term planning models to account for the operational flexibility in power systems planning by analyzing the differences in generation mixes, system costs, curtailment levels, or a combination of these. None of those studies, however, have performed a quantitative assessment using metrics that are specifically designed to evaluate the operational flexibility aspect. Furthermore, the mentioned planning models are computationally intensive, so that different ad-hoc methods for the horizon reduction have been used but neglecting to address the bias that these could introduce on the results of the assessment.

To overcome these limitations, in this paper we introduce an integrated operational flexibility assessment framework that (i) is based on consistent horizon reduction methods driven by an explicit optimization objective to avoid biases that can arise from ad-hoc approximations and (ii) adopts suitable metrics to quantitatively assess the flexibility level of the obtained plans.

Within the framework presented, an integrated GEP-UC model is proposed, cast as a mixed integer linear programming (MILP) problem, and we employ the integer clustering method for handling discrete decision variables [21], which provides a good approximation and a significant reduction of the computational complexity. A 10 years planning horizon is considered based on historical load and RES capacity factor data obtained for the national system of France. The horizon reduction is systematically dealt with by the implementation of a dynamic programming algorithm that optimizes the step-function approximation of the traditional GEP model and an exhaustive search algorithm for the chronological load approximation of the integrated GEP-UC model. For the quantitative operational flexibility assessment, the probabilistic metric of insufficient ramping resource expectation (IRRE) proposed in [9] is used. We complement this measure by originally introducing the expected flexibility shortfall (EFS) metric, which indicates the expected load loss when the system is not able to adequately respond to the inter-temporal variability.

The numerical examples consider a single-region green-field planning problem with no generation units existing in the system. On one hand, this is done to avoid any bias those existing units may impose on the expansion plans and, therefore, to focus solely on the models outcomes; on the other hand, it is done to validate the framework capability for efficiently addressing large-sized instances. The framework, however, is straightforwardly applicable to grey-field planning problems and easily extendable to muli-regional planning. A wide range of RES penetration levels ( $0 \%$ to $50 \%$ ), most notably wind and solar, is considered and the same for carbon emission limits.

The original contributions of the work are:

- The paper contributes to power systems planning with high shares of RES penetration and stringent carbon targets, by proposing a computationally efficient, multi-period integrated GEP-UC model that accounts for key short-term constraints and chronological net load representation. In particular,

* The importance of considering these constraints to account for operational flexibility under high RES penetration has been demonstrated quantitatively by comparing the output of the integrated model to that of the traditional GEP, which leads to investment decisions based on average system operating conditions.

* For computational tractability, horizon reduction is introduced by systematic optimization, to avoid biases on the results obtained by ad-hoc methods.

- The paper introduces the expected flexibility shortfall (EFS) metric for operational flexibility assessment to capture the expected amount of load loss specifically due to insufficient flexibility. We analyze the complementarity of this metric to other metrics of literature, most notably the insufficient ramping resource expectation (IRRE) proposed in [9] which considers the expected frequency of flexibility shortage rather than its magnitude.

- The relevance of the overall modeling for real applications is shown by considering a case study representing the national system of France, with load and RES capacity-factor data spanning a 10-years planning period. Sensitivity to key supply and demand parameters is also performed.

- The results of the paper for a wide range of RES penetration targets and carbon emission limits allows highlighting the importance of 
relying on suitable metrics for the assessment rather than on quantities typically considered for power system planning, like generation mixes, system costs and amount of renewable curtailment, which are not capable to reflect the true flexibility levels of the obtained plans.

For real applications, the integrated framework can be used by power system planners to rapidly and accurately evaluate the impact of different system parameters and policy requirements on the resulting generation expansion plans, most notably in terms of operational flexibility. The planner can, then, adapt the policy requirements to ensure generation plans with an adequate flexibility level or set proper expectations on which levels are attainable under a specific set of parameters and requirements. Moreover, several remarks are highlighted in the relevant sections regarding the proper treatment of RES investments as decision variables within the simplified optimization model as well as the horizon reduction method, which can prove useful for practitioners if similar types of models are to be considered.

\section{Operational flexibility assessment framework}

\subsection{Overview}

The integrated operational flexibility assessment framework proposed is schematically illustrated in Fig. 1. For comparison purposes, it will be applied to two types of power system planning models: the traditional GEP model soft-linked with a UC model (denoted S-GEP) and the integrated GEP-UC model solved as a single optimization (denoted C-GEP). Fig. 1a illustrates the assessment framework applied to the former, and Fig. $1 \mathrm{~b}$ that applied to the latter. The framework stands: (i) the formulation of the models, (ii) the elaboration of accurate horizon reduction approximation methods and (iii) the elaboration of suitable metrics for assessment of the operational flexibility of the obtained plans. The schematic illustration summarizes the different steps

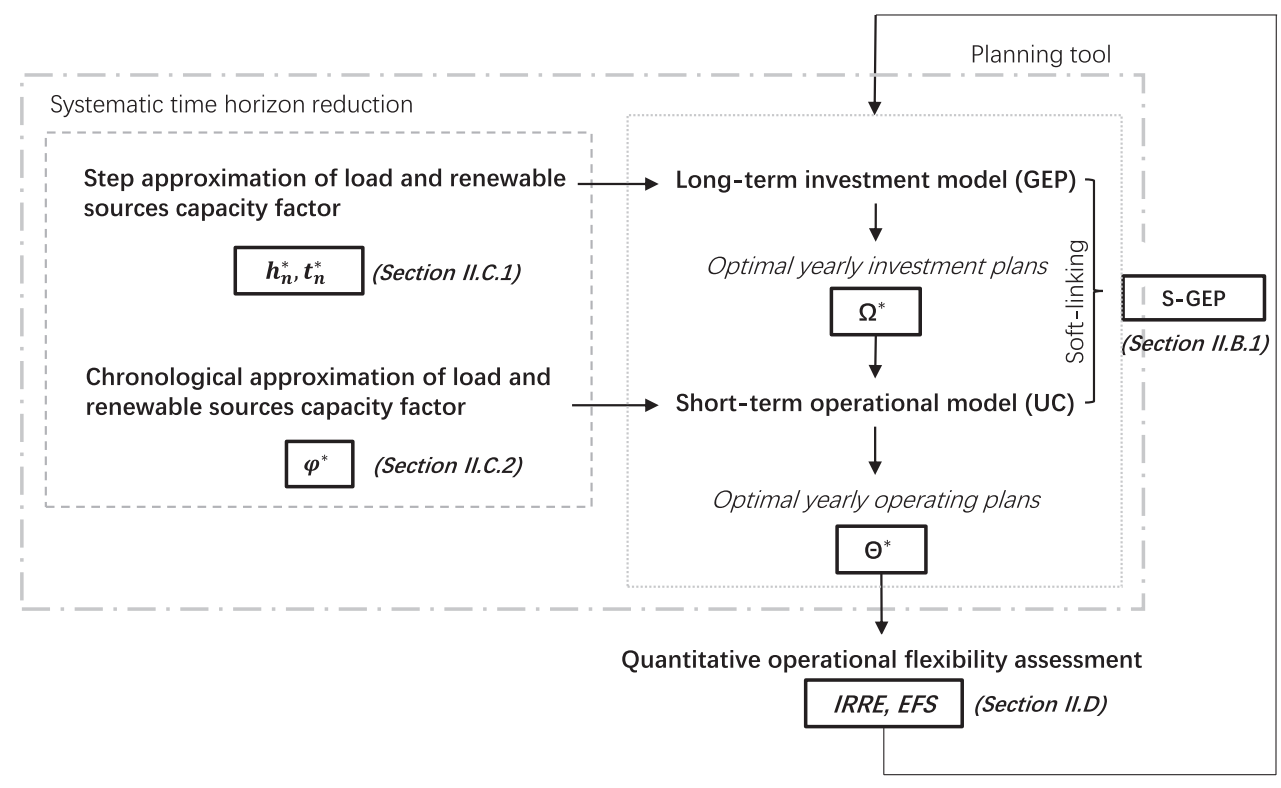

(a) Soft-linked planning models

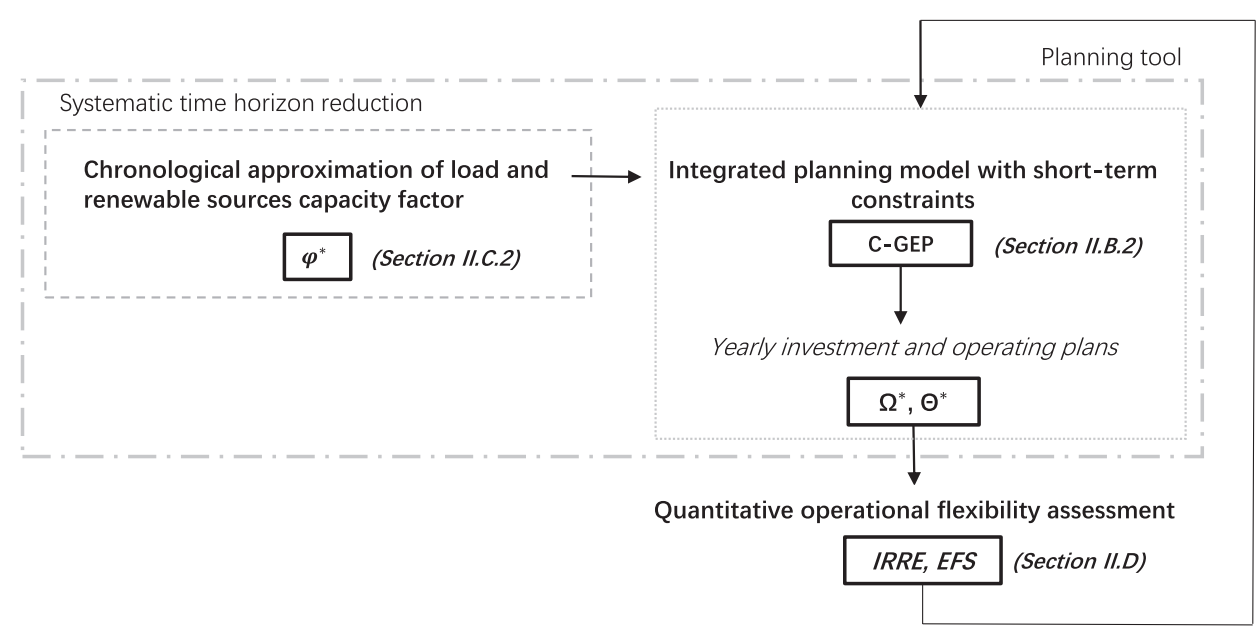

(b) Integrated models

Fig. 1. Operational flexibility assessment framework. 
in applying the framework. The planner would, first, employ the proper systematic horizon reduction method with respect to the planning tool of choice. Optimal generation expansion plans and their operation are, then, obtained through the optimization models. Finally, the defined metrics are used to quantify the operational flexibility levels of the obtained plans. The planner can, then, evaluate whether or not the plans meet the required levels of flexibility and can evaluate the impact of adjusting the different parameters on the plans obtained. Each of these elements is described in details in the following sections.

\subsection{Power system planning models formulation}

As previously mentioned, two types of power system planning models are considered for operational flexibility assessment:

\subsubsection{Soft-linked GEP-UC model (S-GEP)}

The S-GEP model consists of two mixed integer linear programming problems which are successively solved:

i. A long-term GEP model, which has the objective of minimizing the total discounted cost over the planning horizon under typical longterm simplified constraints. No hourly chronological order is considered and load is represented as load blocks derived from a loadduration curve (LDC) with duration (Dury,s) and levels $\left(L_{y, s}\right)$. The detailed model formulation can be found in Appendix A.1

ii. A short-term UC model, which has the objective of minimizing the short-term operational costs, taking into account the detailed shortterm technical capabilities of the units, the chronological demand and RES availability. The detailed model formulation can be found in Appendix A.2

The soft linking of the two problems is achieved by:

1. Solving the long-term GEP problem first, under the simplified system representation;

2. Populating the obtained investment plans within the UC problem, which is, then, solved to obtain the detailed operation of these plans.

\subsubsection{Combined GEP-UC model (C-GEP)}

The C-GEP model is the straightforward integration of the two models described above into a single optimization model, with the following adjustments:

- The load-duration step representation indexed by $(s)$ in the S-GEP model is replaced by the hourly chronological representation $(j)$, for all parameters and decision variables.

- The detailed operating cost equation of the UC objective function replaces that of the traditional GEP model.

The resulting high resolution MILP problem is known to be computationally challenging, especially when considering a multi-annual planning horizon: (i) the formulation has been, therefore, adapted to handle discrete decision variables by means of the integer clustering method proposed in [21]; (ii) and time horizon reduction is introduced systematically, as explained in the next section.

\subsection{Time horizon reduction}

The traditional GEP, UC and integrated GEP-UC models have different natures, notably in the time resolution considered for the load and RES capacity factor (CF). Different approximation methods are, therefore, employed for the horizon reduction.

\subsubsection{Load and RES-CF approximations for the GEP model}

To obtain the investment decisions in the traditional GEP model, yearly load is typically represented as a load-duration curve (LDC), which is approximated by a step-function, where each step represents an average load-level and duration. This function is typically obtained by deciding in an ad-hoc manner the number of steps and segmenting the LDC accordingly. Depending on the choice of segmentation, the outcome of the model can greatly differ, for instance, if more steps are introduced for the peak load hours or the base ones. Moreover, in a multi-annual planning context the LDC forecast varies among the different years, so that a segmentation strategy for a year might not be optimal for other years. A consistent method for the LDC approximation is, therefore, necessary.

The seminal work of [22] is one of very few studies in power systems planning found to address this issue. We define a similar optimization problem where the objective of the step function approximation is the minimization of the energy mismatch between each approximated step and its actual corresponding segment. The optimization problem can be formulated as:

$\min _{\mathbf{t}} \sum_{n=1}^{N} \sum_{i=t_{n-1}}^{t_{n}}\left(F(i)-h_{n}\right)^{2}$

subject to:

$h_{n}=\frac{1}{t_{n}-t_{n-1}} \cdot \sum_{i=t_{n-1}}^{t_{n}} F(i), \quad n=1,2, \ldots, N$

where $F(i)$ represents the actual LDC function, $N$ is the total number of steps specified for the approximation, $n$ is the index of the current approximation step, $h_{n}$ is the height (load level) of the step function for step $n$ and $t_{n}$ is the breakpoint at which the step function changes value from step $n$ to step $n+1$. The objective is to find the breakpoints vector $t$ which fully defines the approximated step function and is such to minimize the mismatch of Eq. (1). This problem can be stated as a dynamic programming problem, where the backward recursive functional equation is defined as:

$f_{n}(x)=\min _{x \leqslant t \leqslant T}\left[\sum_{i=x}^{t}\left(F(i)-h_{N-n+1}\right)^{2}+f_{n-1}(t)\right], \quad n=1,2, \ldots, N$

for which $h_{N-n+1}$ can be calculated as given in Eq. (2).

It is important to note that since we consider the investment in RES capacity as a decision variable, it heavily depends on the average capacity factor (CF) of each RES technology. Neglecting this consideration within the simplified long-term GEP problem is equivalent to assuming that wind and solar technologies are fully dispatchable. Constraint (A.11) in the Appendix is, therefore, introduced to avoid this inaccuracy. Similar to the LDC approximation, the CF of each RES technology can be approximated by a level and a duration. However, the real average correlation between the load and the RES-CF should be maintained to avoid unrealistic and biased results. An illustration of the approach followed in this work to achieve this is given in Appendix B.

\subsubsection{Load and RES-CF approximations for the UC and GEP-UC models}

The horizon reduction for the C-GEP and UC models need to maintain the real hourly chronological order of both the load and the RES-CF. This is typically achieved by approximating the full year to a number of days, weeks or months while preserving the sub-period chronological order. We opt to represent a year by a number of sampled weeks obtained by solving an optimization problem similar to that proposed by De Sisternes and Webster [23], which reported superior approximations than other ad-hoc methods.

The weeks are selected with the objective of minimizing the energy mismatch between the actual LDC and the approximated one ( $L D C^{a p r o x}$ ) obtained through scaling up the weeks sampled to the full year length. Mathematically this is represented by [23]:

$\phi^{*} \in \underset{\phi}{\operatorname{argmin}} \sum_{i=0}^{T}\left(L D C_{i}-L D C_{\phi, i}^{a p r o x}\right)^{2}$ 
where $T$ is the total number of hours in each planning year and $\phi$ is a vector containing the set of indexes of the selected weeks. The optimization can be solved using an exhaustive search that evaluates all possible combinations of the $n$ specified weeks and selects those that minimizes the energy mismatch. Once the optimal weeks are obtained for the load, the same weeks are selected for the RES-CF data to ensure that the correlation between the two is maintained.

\subsection{Flexibility assessment metrics}

To quantify the flexibility of the system, we adopt two probabilistic metrics: the insufficient ramping resource expectation (IRRE) proposed in [9], and the originally introduced expected flexibility shortfall (EFS) metric.

\subsubsection{Insufficient Ramping Resource Expectation (IRRE)}

The IRRE is the expected number of instances in which the generation units in a power system cannot answer to the changes in net load. The metric is generally obtained by [9]:

1. Calculating the net load ramping time series for the whole planning horizon in both upwards (up) and downwards (dn) directions.

2. Calculating the up/dn available flexible resources within a specified time horizon of interest (e.g. one hour), given the availability and commitment status of each generation unit, its start-up time, its actual production level and its total upwards or downwards ramping capabilities for the next period.

3. Aggregating all the time series for all resources to obtain the total up/dn available flexibility time series.

4. Calculating the up/dn available flexibility empirical cumulative distribution function from the total available flexibility time series.

5. Calculating the probability of insufficient ramping by substituting the required net load ramping in the obtained distribution function. The sum of the up/down probabilities time series gives the IRRE $+/-$.

\subsubsection{Expected Flexibility Shortfall (EFS)}

While the IRRE indicates the expected frequency for not meeting the flexibility requirements, it does not give any information about how short the system is on average when not able to meet these requirements. This can be calculated through the expected flexibility shortfall (EFS) metric.

The EFS metric builds on the value-at-risk (VaR) measure defined as the "possible maximum loss over a given holding period within a fixed confidence level" [24]. Mathematically, this is defined as:

$V A R_{\alpha}(X)=\sup \{x \mid P[X \geqslant x]>\alpha\}$

where $X$ in our context is a variable denoting the loss of load due to insufficient flexibility and $\sup \{x \mid P[X \geqslant x]>\alpha\}$ indicates the highest $100 \alpha$ percentile of the loss distribution.

The expected flexibility shortfall (EFS) is, thus, the conditional expectation of load loss due to insufficient flexibility, given that it is beyond the VaR level, or:
$\operatorname{EFS}_{\alpha}(X)=E\left[X \mid X \geqslant \operatorname{VaR}_{\alpha}(X)\right]$

The EFS is calculated by performing steps (1)-(3) of the IRRE calculations, followed by:

4. Calculating the up/dn losses time series as the absolute difference between the up/dn net load ramping series and the respective total available flexibility resources.

5. Calculating the VaR at the desired $100(1-\alpha) \%$ confidence levels.

6. Calculating the EFS as the average loss for observations exceeding the VaR level, at the respective confidence levels.

\section{Numerical example}

\subsection{Test system}

For the multi-annual demand representation, we have taken the 10 years load data of France, from 2006 to 2015, which are publicly available at [25], to represent a realistic system demand for 10 planning years. We have similarly calculated the RES-CF, namely wind and solar power, from the actual yearly production time series, by dividing each hourly production by the total installed capacity of each technology. This results in the hourly CF time series per renewable technology and for each year.

Table 1 summarizes the technical and cost data for the generation technologies considered in the expansion planning. The cost data and units capacities are obtained from the IEA/NEA Projected Costs of Generating Electricity report (2015 edition) [26]; the remaining technical characteristics are largely based on data described in [27] to maintain consistency with characteristics relevant to the French power system. The discount rate is assumed to be $3 \%$, the minimum design reserve margin $r^{\min }$ is set to $15 \%$ of the maximum annual load, operating reserves are set to cover $1 \%$ of the hourly load for primary and secondary reserves, and $10 \%$ of the hourly RES production for upwards and downwards secondary reserves. The penalty for not meeting demand $\left(C^{l n s}\right)$ is set to $4 \mathrm{k} € / \mathrm{MWh}$ to discourage load shedding. Finally, the construction time of new units is neglected, as we are considering a relatively concise planning horizon.

\subsection{Implementation notes and remarks}

All optimization problems are modeled in the Python programming language. The MILP problems are programmed using the Pyomo software package $[28,29]$ and solved using IBM ILOG-CPLEX with an optimality gap of $0.1 \%$. It is important to note that for the UC and the GEP-UC models, the yearly demand is approximated by 4 representative weeks and the chronological order within each week is maintained; however, the immediate demand profile change between one week and the next may not reflect the realistic variation that could occur in the system. It is important, therefore, to decouple the operational decision variables from one week to the next, to eliminate any bias from incorrect initialization. In this respect, unit states are constrained to be identical at the beginning and end of each week, which reasonably assumes that each week is followed by a similar one.

Table 1

Technical and economic characteristics for the different generation technologies.

\begin{tabular}{|c|c|c|c|c|c|c|c|c|c|c|c|}
\hline $\begin{array}{l}\text { Technology } \\
{[i]}\end{array}$ & $\begin{array}{l}P_{i}^{\max } \\
{[\mathrm{MW}]}\end{array}$ & $\begin{array}{l}P_{i}^{\min } \\
{[\mathrm{MW}]}\end{array}$ & $\begin{array}{c}R_{i}^{U \max } \\
{[\mathrm{MWh} / \mathrm{min}]}\end{array}$ & $\begin{array}{c}R_{i}^{D \max } \\
{[\mathrm{MWh} / \mathrm{min}]}\end{array}$ & $\begin{array}{l}M_{i}^{u} \\
{[\mathrm{~h}]}\end{array}$ & $\begin{array}{l}M_{i}^{d} \\
{[\mathrm{~h}]}\end{array}$ & $\begin{array}{l}E_{i} \\
{\left[\mathrm{tCO}_{2} / \mathrm{MWh}\right]}\end{array}$ & $E F O R_{i}$ & $\begin{array}{l}C_{i}^{i n v} \\
{[\mathrm{M} €]}\end{array}$ & $\begin{array}{l}C_{i}^{m r g l} \\
{[€ / \mathrm{MWh}]}\end{array}$ & $\begin{array}{l}C_{i}^{S} \\
{[\mathrm{k} €]}\end{array}$ \\
\hline Nuclear & 1400 & 700 & $0.5 \% P_{n} / \mathrm{min}$ & $0.5 \% P_{n} / \mathrm{min}$ & 12 & 48 & 0 & 0.01 & 3.95 & 9.33 & 15.0 \\
\hline Fossil hard coal & 1100 & 550 & $1.5 \% P_{n} / \mathrm{min}$ & $1.5 \% P_{n} / \mathrm{min}$ & 6 & 10 & 0.96 & 0.06 & 2.08 & 36.67 & 11.26 \\
\hline Fossil gas (CCGT) & 550 & 165 & $5 \% P_{n} / \min$ & $5 \% P_{n} / \min$ & 3 & 5 & 0.46 & 0.04 & 1.02 & 69.00 & 7.53 \\
\hline Fossil gas (OCGT) & 270 & 54 & $20 \% P_{n} / \min$ & $20 \% P_{n} / \mathrm{min}$ & 1 & 2 & 0.67 & 0.08 & 0.7 & 110.00 & 3.79 \\
\hline On-shore wind & 80 & 0 & / & / & / & / & 0 & $*$ & 1.9 & 0 & / \\
\hline Solar-PV & 60 & 0 & / & / & / & / & 0 & $*$ & 1.5 & 0 & / \\
\hline
\end{tabular}




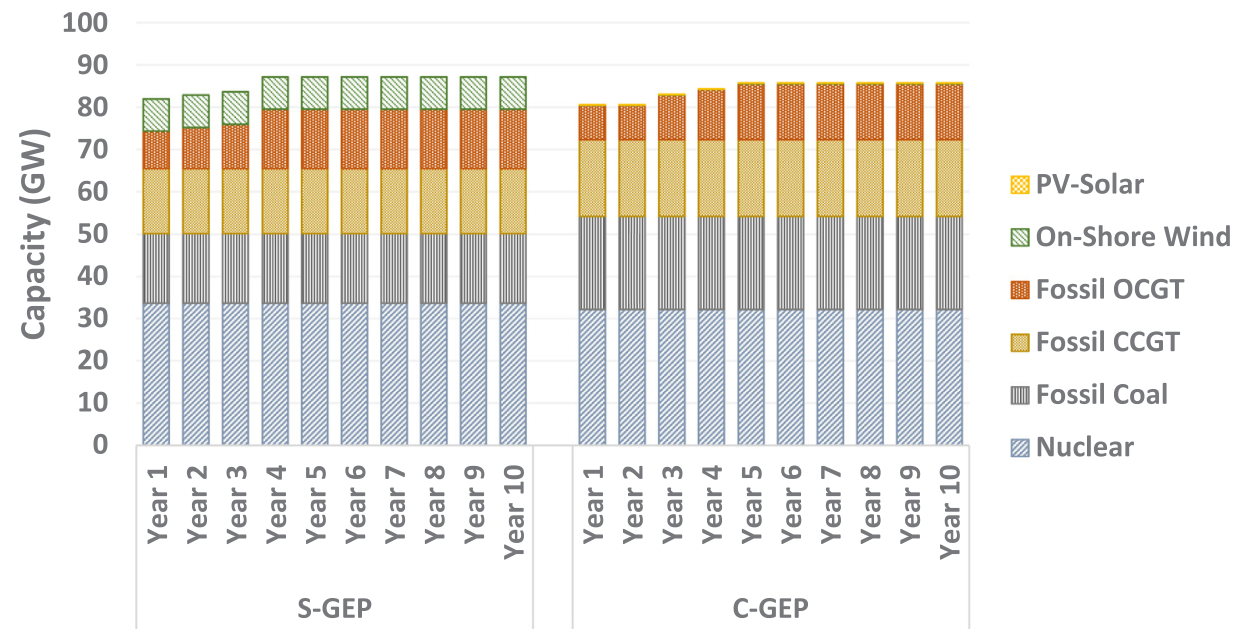

Fig. 2. Cumulative yearly installed capacity for the base case obtained through the S-GEP (left) and C-GEP (right) models.

\section{Results and discussion}

\subsection{RES penetration and carbon emission policy scenarios}

We first explore 12 scenarios covering a wide range of RES penetration and carbon emission targets: a base case with no RES nor emission requirements, in addition to all remaining combinations of $0 \%, 25 \%, 35 \%$ and $50 \%$ binding RES penetration targets (as a percentage of total electricity demand) and $0 \%, 75 \%$ and $50 \%$ emission limit (as a percentage of each corresponding no emission limit scenario). We apply the assessment framework on the two types of planning models considered (C-GEP and S-GEP), for comparing the effect of integrating the short-term constraints within the long-term investment planning problem, primarily in terms of operational flexibility. For the S-GEP model, each annual LDC is approximated by twelve load-duration steps using the dynamic programming optimization described in Section 2.3.1. The normalized root mean squared error (NRMSE) of the energy mismatch for the ten approximated yearly LDC have a mean of $3.91 \%$ and a standard deviation of 0.002. For the C-GEP model, each year is approximated by four weeks through the optimization problem described in Section 2.3.2, where within each week, hourly chronological order is maintained. The NRMSE of the 10 approximated yearly loads have a mean of $0.63 \%$ and a standard deviation of 0.003 .

\subsubsection{Base case}

We start by investigating the results of the base case for both S-GEP and C-GEP models. Fig. 2 illustrates the total capacity installed of each generation technology, at the end of each year as obtained by each model. The bulk of the investments is done in the first year, where $82.01 \mathrm{GW}$ and $80.59 \mathrm{GW}$ total capacities are installed as given by the SGEP and the C-GEP model, respectively. These capacities gradually increase to the end of the planning horizon. The final total capacities installed are $87.14 \mathrm{GW}$ and $85.72 \mathrm{GW}$ for S-GEP and C-GEP, respectively. The additional investments in both cases are in the Fossil OCGT technology. It can be observed that, in this case of no RES penetration requirement, the capacity investments given by both models closely match (see Fig. 2).

To further assess the S-GEP and C-GEP obtained plans, a number of performance measures are compared. We consider the amount of load not served (LNS), the amount of RES shedding, the total carbon emission and the total cost (investment + operating costs) ${ }^{2}$ of the plans obtained through each model for the whole planning horizon. Table 2a summarizes the results of the different measures for the base case. The

\footnotetext{
${ }^{2}$ Excluding the cost of LNS to avoid redundancy.
}

C-GEP model outperforms the S-GEP in the resulting LNS, amounting to $0.01 \%$ of the total load for the former as opposed to $0.12 \%$ for the latter. Since only a small fraction of the capacities installed are in RES technologies, no RES shedding was needed. The increased inter-temporal variability caused by large RES production could, typically, make it more cost effective to shed higher quantities of renewable energy than to cycle thermal units. For carbon emission, operating the C-GEP plan results in higher total emission $(\approx 1700$ Mtons compared to $\approx 1380$ Mtons by the S-GEP model) since more fossil capacities are installed. The total investment and operating cost is lower for the C-GEP plan $(310.59 \mathrm{Bn} €)$ compared to the S-GEP one $(313.00 \mathrm{Bn} €)$. This, together with the lower amount of LNS, highlights that the C-GEP plan is better adapted to satisfy the load at lower cost.

The quantitative operational flexibility assessment of the resulting plans is summarized in Table $2 \mathrm{~b}$. The IRRE and EFS results are reported for the total upwards $(+)$ and downwards $(-)$ net load variations. The results show that operating the S-GEP plan is expected not to satisfy $7.38 \%$ of the total number of upwards ramps (IRRE+) as opposed to $2.20 \%$ for the C-GEP plan. Similarly, the EFS + is higher for the S-GEP plan, amounting to $512.00 \mathrm{MW}$ and $2400 \mathrm{MW}$ compared to $90 \mathrm{MW}$ and $450 \mathrm{MW}$ for the C-GEP plan at the $95 \%$ and the $99 \%$ confidence intervals, respectively. For the negative ramps, it is shown that the operational flexibility shortages are of much less significance, albeit still being slightly worse for the S-GEP plan.

\subsubsection{Increasing RES penetration and emission limit policies}

Table 3 summarizes the results of the different performance measures for the base-case (top-left corner), along with the different combinations of RES penetration (horizontally) and emission limit (vertically) policies considered. First, we notice how an increased RES penetration requirement leads to a significant difference in the final capacity mix given by the S-GEP and the C-GEP models. Moving horizontally across the Table, i.e. to higher RES penetration, the "shortterm aware" C-GEP model results in mixes with higher fossil capacities and much less nuclear than the S-GEP one. Fossil technologies possess overall better short-term dynamic properties, such as faster ramping and cycling capabilities. The investment decisions derived by the integrated C-GEP model, therefore, accounts for the increased short-term variability by shifting the mix towards these technologies. Moreover, the total installed capacities are higher for the C-GEP plans compared to the S-GEP ones. This difference increases for higher RES penetration, from around $10 \mathrm{GW}$ (for the 25\% RES penetration scenario) up to 30 GW (for the 50\% RES penetration scenarios). This is since the S-GEP model overestimates the actual RES availability as it considers average capacity factor values, resulting in lower perceived need for total capacity investments. 
Table 2

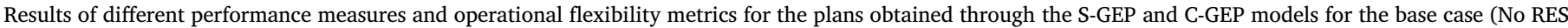
requirement, no carbon limits) (worse performance highlighted).

\begin{tabular}{lcccc}
\hline & \multicolumn{3}{c}{$\begin{array}{c}\text { (a) Installed capacities and generic performance measures results } \\
\text { C-GEP }\end{array}$} & Difference [\%] \\
\cline { 1 - 3 } Total RES Installed Capacity [GW] & 7.60 & 0.14 & (C-GEP relative to S-GEP) \\
Total Installed Capacity [GW] & 87.14 & 85.72 & (C-GEP & $-0.77 \%$ \\
\hline Total Cost (excluding LNS) [B€] & 313.00 & 310.59 & $-89.38 \%$ \\
Load Not Served [\% of total load] & $0.12 \%$ & $0.01 \%$ & $/$ \\
RES shedding [\% of total RES] & $0.00 \%$ & $0.00 \%$ & $+23.22 \%$ \\
Carbon Emission [Mtons] & 1379.60 & 1699.93 & \\
\hline
\end{tabular}

Difference [\%]

S-GEP C-GEP (C-GEP relative to S-GEP)

(b) Operational flexibility metrics results

\begin{tabular}{|ccccc}
\hline IRRE+ (\% of upwards ramps) & $7.38 \%$ & $2.20 \%$ & $-70.13 \%$ \\
\hline \multirow{2}{*}{ EFS+ (MW) } & EFS+ (95\%) & 512.70 & 90.53 & $-82.22 \%$ \\
& EFS+ (99\%) & 2402.85 & 451.31 & $-38.08 \%$ \\
\hline \multirow{2}{*}{ IRRE- (\% of downwards ramps) } & $0.01 \%$ & $0.00 \%$ & $-42.52 \%$ \\
\hline \multirow{2}{*}{ EFS- (MW) } & EFS- (95\%) & 0.00 & 0.00 & $/$ \\
& EFS- (99\%) & 0.00 & 0.00 & $/$ \\
\hline
\end{tabular}

Table 3

Results of the different performance measures for the plans obtained through the S-GEP and C-GEP models for the range of RES penetration and carbon emission limits considered (worse performance highlighted)

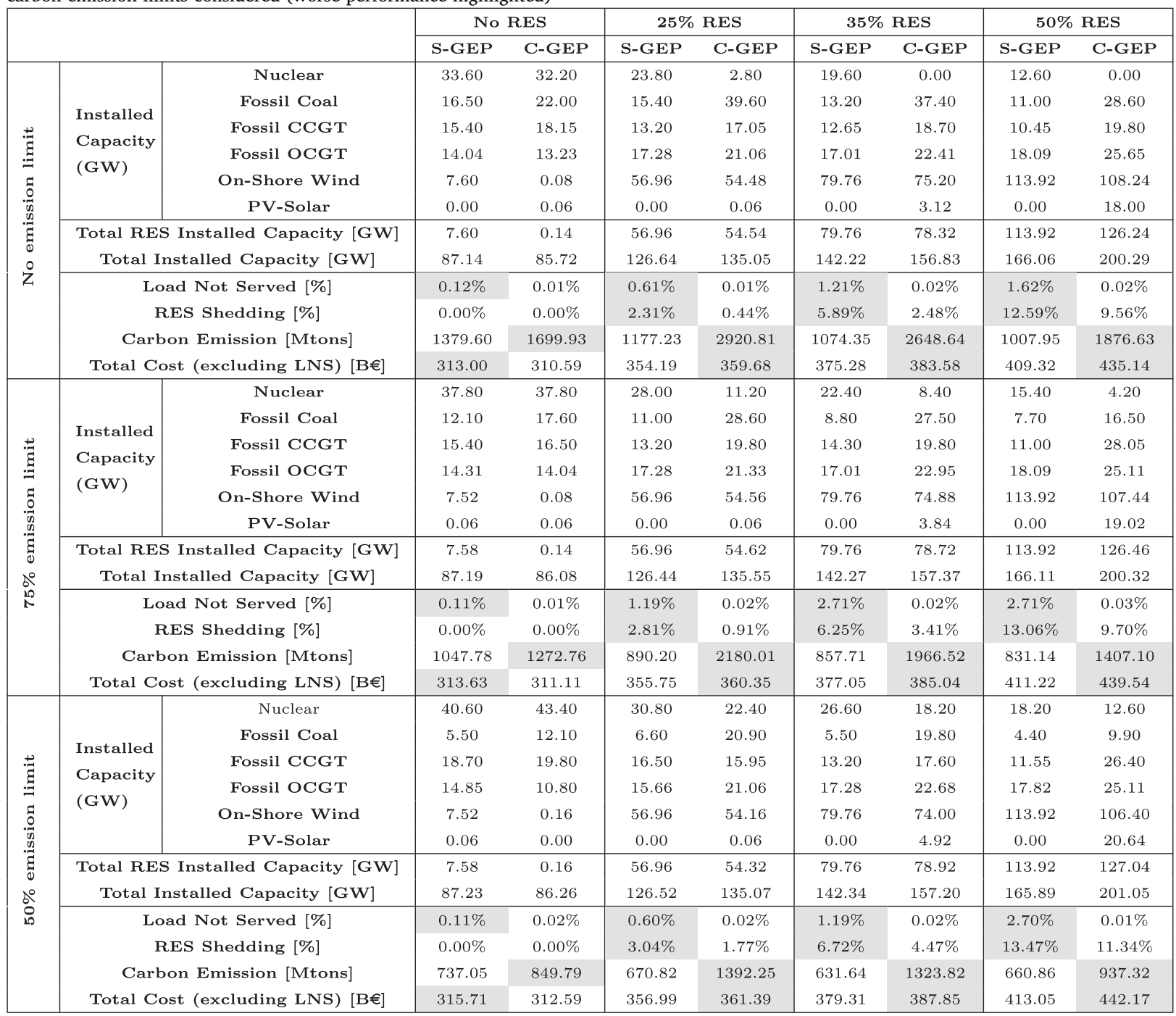


The impact of these capacity differences is reflected in the resulting LNS percentage. There is a steep increase in the amount of LNS for the S-GEP plans, from $0.12 \%$ and up to $2.7 \%$ for the highest RES penetration scenarios. This represents an unacceptably high level of LNS and defies the security of supply of the obtained plans. The C-GEP plans, on the other hand, maintain a low average LNS percentage of $0.02 \%$, with negligible variation across all scenarios.

RES shedding is shown to also increase under higher RES penetration levels; however, it remains lower for the C-GEP plans compared to the S-GEP ones. As previously explained, shedding decisions are taken when they are more cost effective than cycling thermal units. The CGEP model accounts for these quantities shed and ensures that the plans obtained, when operated, would still meet the RES quota requirements. This is not the case for the S-GEP model, which may result in final mixes that are theoretically meeting these requirements, but practically are not.

It is shown that under RES penetration scenarios, the C-GEP plans have a higher total investment and operation costs (excluding LNS cost) than the S-GEP ones. However, the increase in these costs is not in the same order of magnitude of the additional capacities installed and the cost of LNS avoided. The maximum cost difference is $+6.31 \%$ higher for the C-GEP plan compared to the S-GEP one (50\% RES scenarios), whereas the installed capacities is around $+20 \%$ higher. The LNS cost avoided in these cases is significant and if taken into consideration would in fact result in lower overall costs for these plans.

Regarding carbon emission; the C-GEP plans have higher emission levels compared to the S-GEP ones, since their capacity mix is characterized by higher amounts of fossil technologies. As previously explained, the optimal decision to shift the mix towards fossil technologies is due to their improved capability to counterbalance the increase in net-load variations caused by high RES levels. This, in fact, indicates that increasing RES penetration levels by itself does not guarantee lower carbon emission levels in the system, since the rest of the capacities would be required to consist of more dynamic yet more emitting units. In this respect, the S-GEP model is considered to underestimate the actual emission levels under real operation. For our numerical example, these underestimations are found to reach levels up to $60 \%$ for the $35 \%$ RES, no emission limit case.

Imposing carbon emission limits, as shown by moving vertically across the table does not have a significant impact on most of these measures. It does, however, considerably affect the capacity mix obtained by the different models. Evidently, as more stringent carbon limits are imposed, the capacity mix shifts towards less emitting technologies and, most notably, nuclear. Most of the fossil capacity reduction is in the fossil coal capacity since it is the most emitting one; the total capacity installed, however, remains almost constant. Furthermore, despite the large differences in the capacity mixes, the total costs of the plans do not heavily vary, averaging around $+1 \%$ increase in most cases.

The quantitative impact of increased RES penetration and stringent emission limits on the operational flexibility of the expansion plans is summarized in Table 4. For the upwards ramping requirements, the results show that the S-GEP plans become significantly short on flexibility as higher percentages of RES penetrate the system. A linear and steep increasing trend of the flexibility shortage, reaching an IRRE + of up to $47 \%$ (for the $50 \%$ RES case), represents a failure to meet half the number of times the system is expected to provide upwards flexibility. The EFS + similarly exhibits a multifold increase relative to plans with lower RES penetrations (e.g. EFS value of $8157.30 \mathrm{MW}$ compared to 2402.85 MW with $99 \%$ confidence, for the 50\% and 0\% RES cases, respectively). For the C-GEP plans, the different metrics report very low expected shortages compared to the S-GEP ones. The IRRE + does not exceed $2.20 \%$ and remains almost constant at an average of $1.30 \%$ for all cases considered. Similarly, the EFS + of the C-GEP plans does not exceed a comparatively lower values in the order of hundreds of MW at most, for all cases considered.
For the downwards ramping requirements, as with the base case, the flexibility shortages are found to be negligible. This is reasonably justified for single-region planning where, as a consequence of considering all generation units located at the same region, sufficient available resources can reduce their output to answer to downward ramps. However, it is still shown through the IRRE- results that systems with very large RES presence would exhibit some flexibility shortage. Generally, downwards flexibility shortage would become more relevant if multi-regional planning is considered, since the available resources will be limited for each region.

Overall, it is shown that the flexibility shortage of the C-GEP plans remain low and almost constant across the different cases considered, while that of the S-GEP plans are much more significant and highly affected by the RES penetration levels than by carbon emission limits. The consistently low C-GEP shortage values do not only indicate this model superior adequacy in accounting for the different RES and carbon requirements, but that it is also able to fully cope to the variations in the different policy requirements, while ensuring adequate operational flexibility levels.

The shown results are found to be consistent with those of the other studies reviewed. Most notably, in Palmintier and Webster [15] where an overall similar investigation was conducted comparing two similar modeling methods as those presented in this work. Their investigation over a range of RES and carbon levels revealed the same trends in the capacity mixes obtained, most notably, that with higher RES penetration, the mix shifts to include more units with faster dynamic properties (typically fossil peaking units). They also showed that carbon emission can be underestimated by $30-60 \%$ by planning models that do not consider short-term system representation. However, they considered only a single-period optimization problem with wind penetration as an exogenous parameter, and did not consider quantitative metrics for the operational flexibility assessment. The multi-period planning considered here allows more realistic planning paradigms, where investment decisions can be optimally taken at different periods, and allows covering a wide variation in the system parameters at the different periods (see for example the wide spectrum of inter-temporal load variations of the four weeks sampled per year in Appendix C). Moreover, the results indicate, that although the capacity mixes may give some indication of the flexibility levels of the plans obtained, they do not capture to what extent these plans are short on operational flexibility. This becomes clear when suitable quantitative metrics are used, as shown in these results.

\subsection{Exploring the effect of fuel cost variation on operational flexibility}

The investment plans obtained are evidently dependent on the set of system parameters initially chosen. Since we consider a deterministic problem, the variation in those parameters could admittedly alter the results obtained, most notably, the uncertainties regarding fuel costs and load evolution trend. We, therefore, opt for exploring selected scenarios representing a wide variation in those parameters, and investigating their effect on the operational flexibility levels of the plans obtained. In this section two scenarios of fuel costs (coal and natural gas costs) are explored: $50 \%$ increase and 50\% decrease, to cover a wide variation of the base case, and consistent with the percentages considered in the IEA report for sensitivity analysis [26]. For clarity, only the results of the C-GEP model are reported for the median 35\% RES penetration level. However, all emission limit scenarios are investigated, since it is reasonable to assume that fuel costs could have a higher impact on the plans obtained when combined with stringent emission limits.

The installed capacities for all fuel cost and emission limit scenarios are illustrated in Fig. 3. For the highest fuel cost scenario, much of the coal and - to a lesser extent - CCGT capacities are substituted by the less emitting nuclear units, more so as tighter emission limits are enforced. For the lower prices, the coal capacity is still substituted, but 
Table 4

Results of the operational flexibility metrics for the plans obtained through the S-GEP and C-GEP models for the range of RES penetration and carbon emission limits considered (worse performance highlighted).

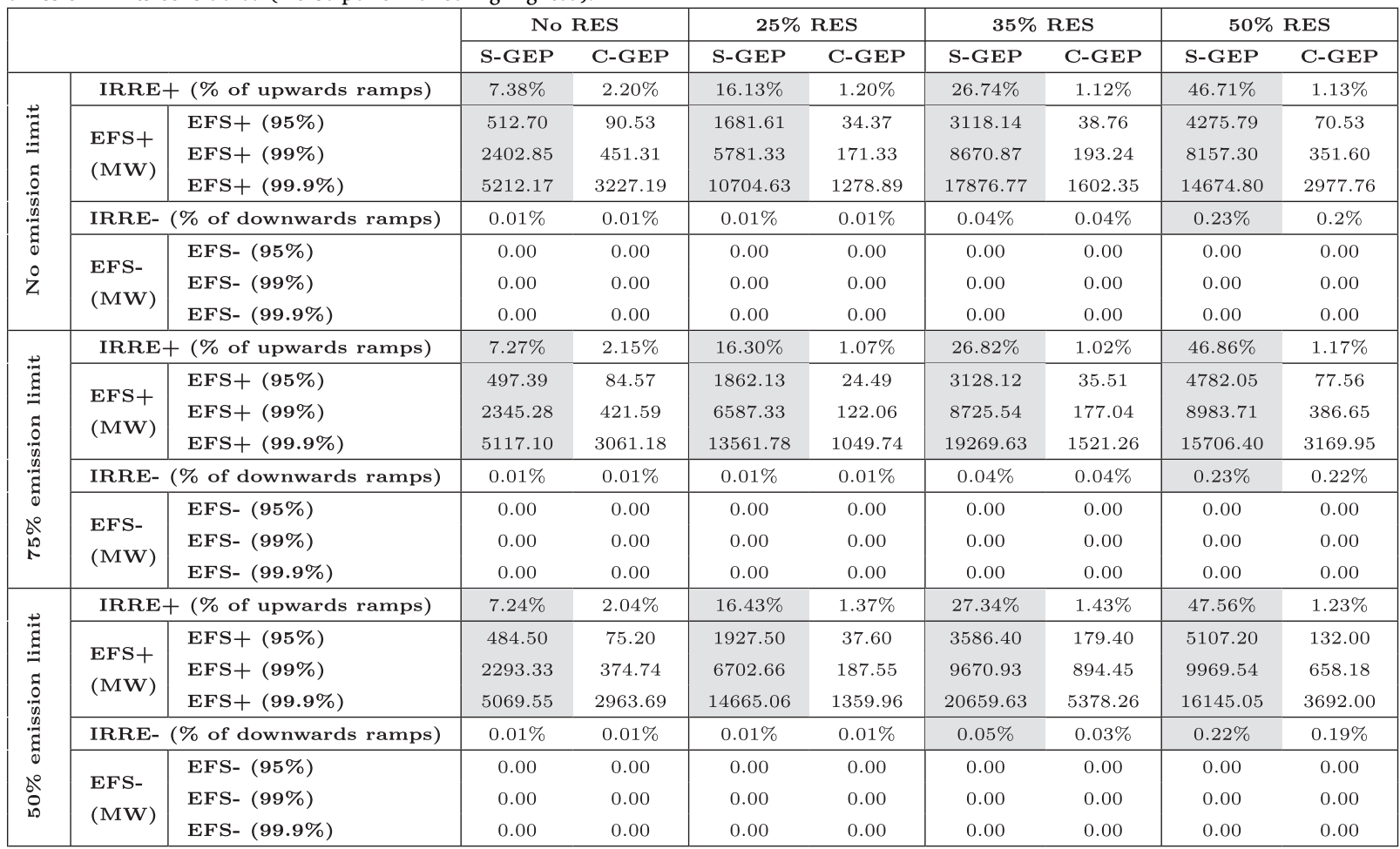

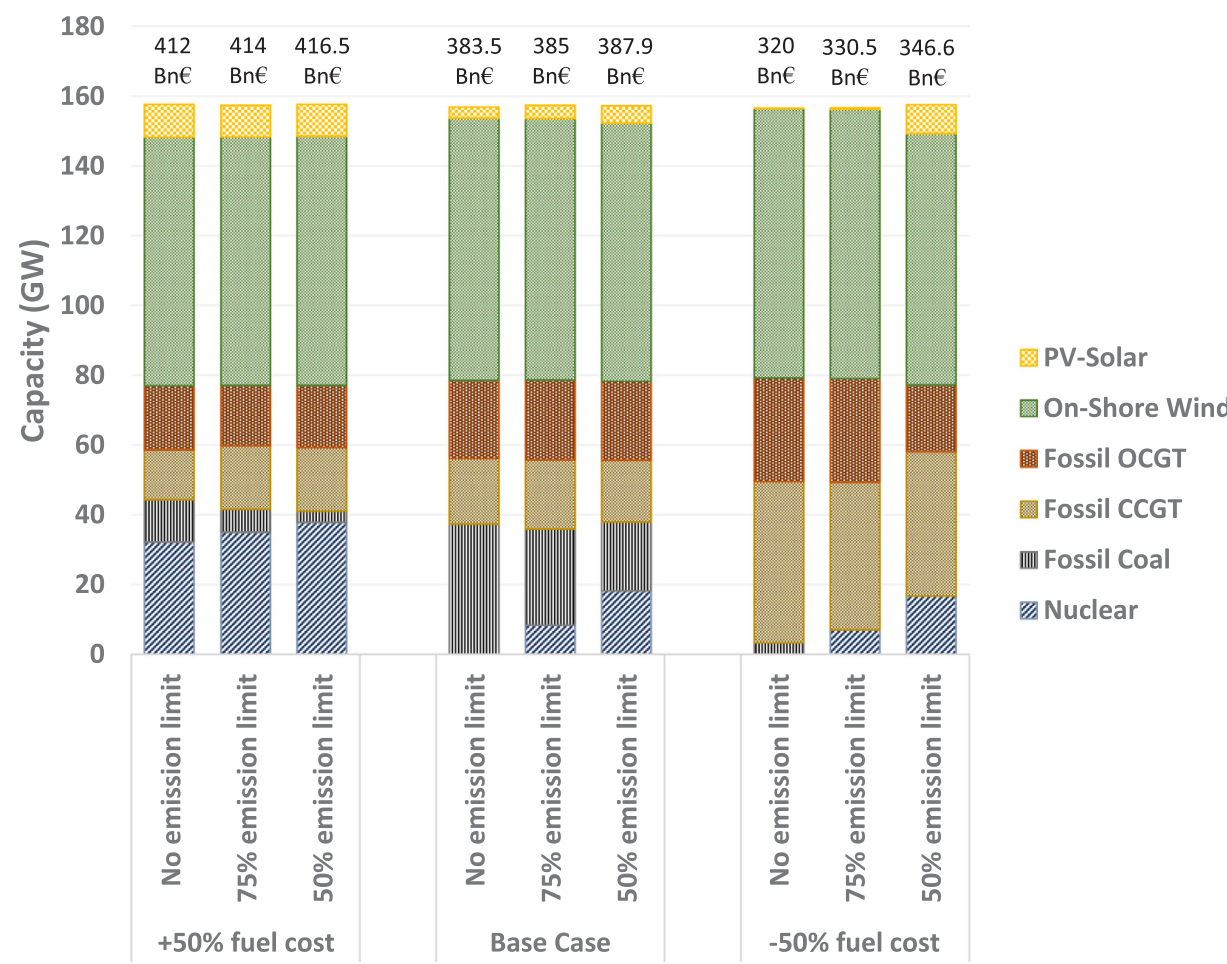

Fig. 3. Total installed capacity as given by the C-GEP model under 35\% RES penetration for the different fuel cost scenarios.

this time by the peaking CCGT and OCGT units. The lower emission requirements are attained through progressive substitution of fossil units by nuclear ones, as can be observed within each fuel cost scenario. The total installed capacity across all scenarios, however, remain constant.

In terms of operational flexibility, Fig. $4 \mathrm{a}$ and $\mathrm{b}$ illustrate the results for the IRRE + and EFS +, respectively. As can be expected, plans obtained under the highest fuel cost have the highest expectation of upwards flexibility shortages. This is because much of the fossil units, which possess better dynamic properties, are replaced by the less flexible nuclear ones. The opposite is observed for the plans obtained at the lowest fuel cost driven by the higher capacities of those peaking 


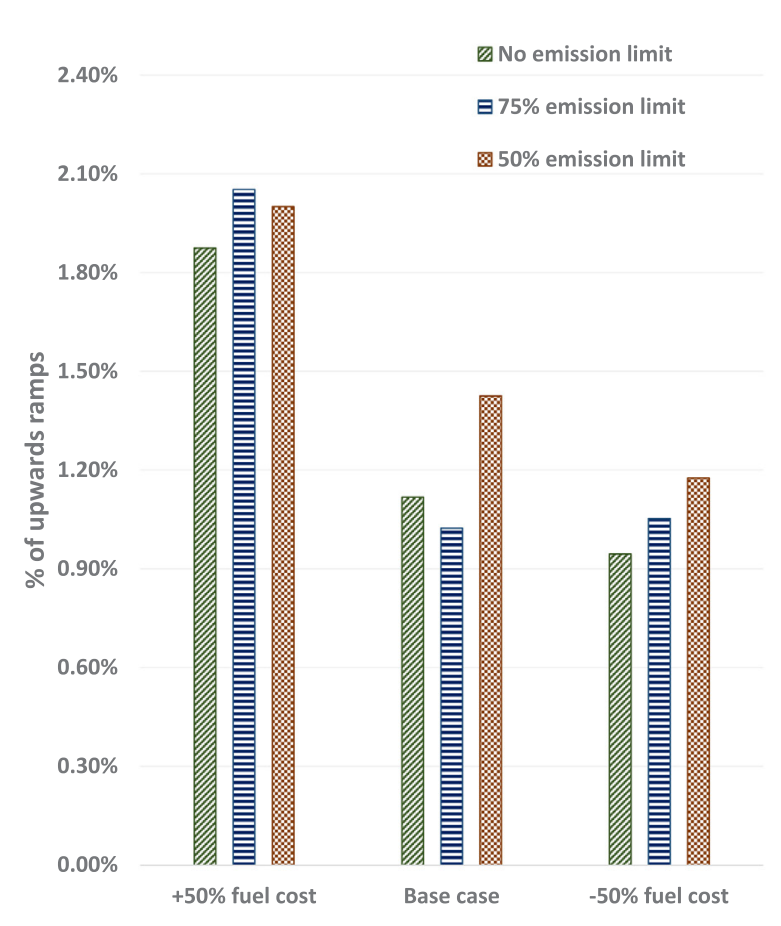

(a) IRRE+

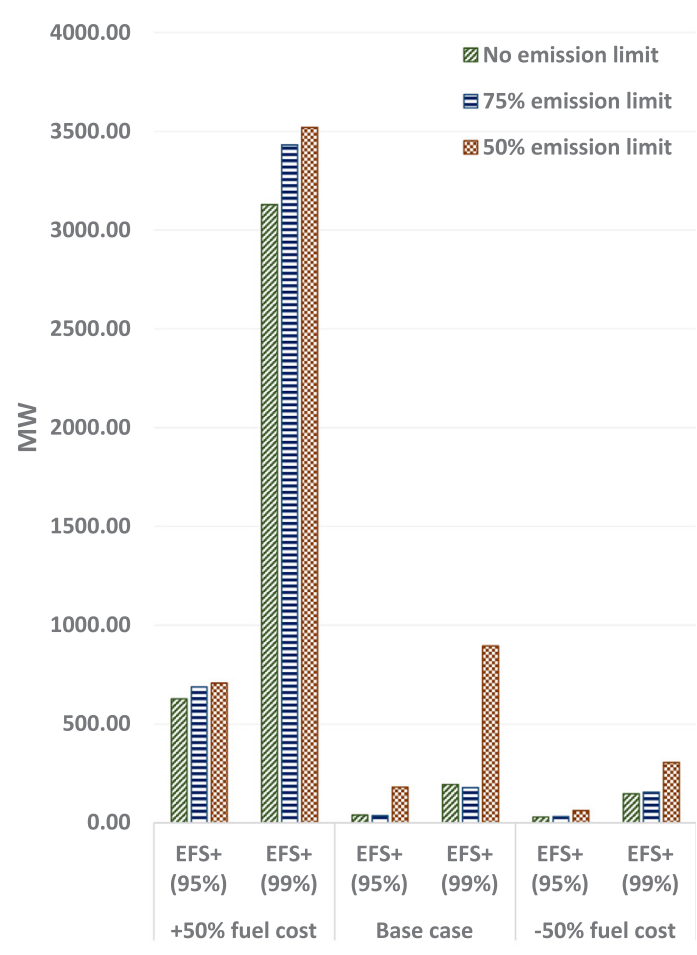

(b) EFS+

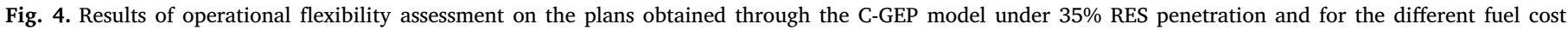
scenarios.

units. Notice, again, that despite this significant variation in the capacity mix across the different fuel scenarios, the IRRE of the integrated CGEP model plans did not exceed $2.05 \%$ of the total number of upwards ramps, with a quasi-linear decreasing trend as a function of less stringent emission limits and decreasing fuel costs. This remains a very small percentage point relative to any shortage value observed for the S-GEP model under RES penetrations. The EFS + confirms the trends observed using the IRRE metric, however, at the highest fuel cost scenario it signaled a relatively high shortage expectations that could go up to the order of several GWs at the $99 \%$ confidence level. Such a magnitude is significant and would be important to account for, and highlights the complementarity of the two measurement approaches for giving an accurate assessment.

\subsection{Exploring the effect of load evolution on operational flexibility}

The load evolution trends could be another source of influence on the plans obtained. In this respect, in their 2016 "Generation Adequacy

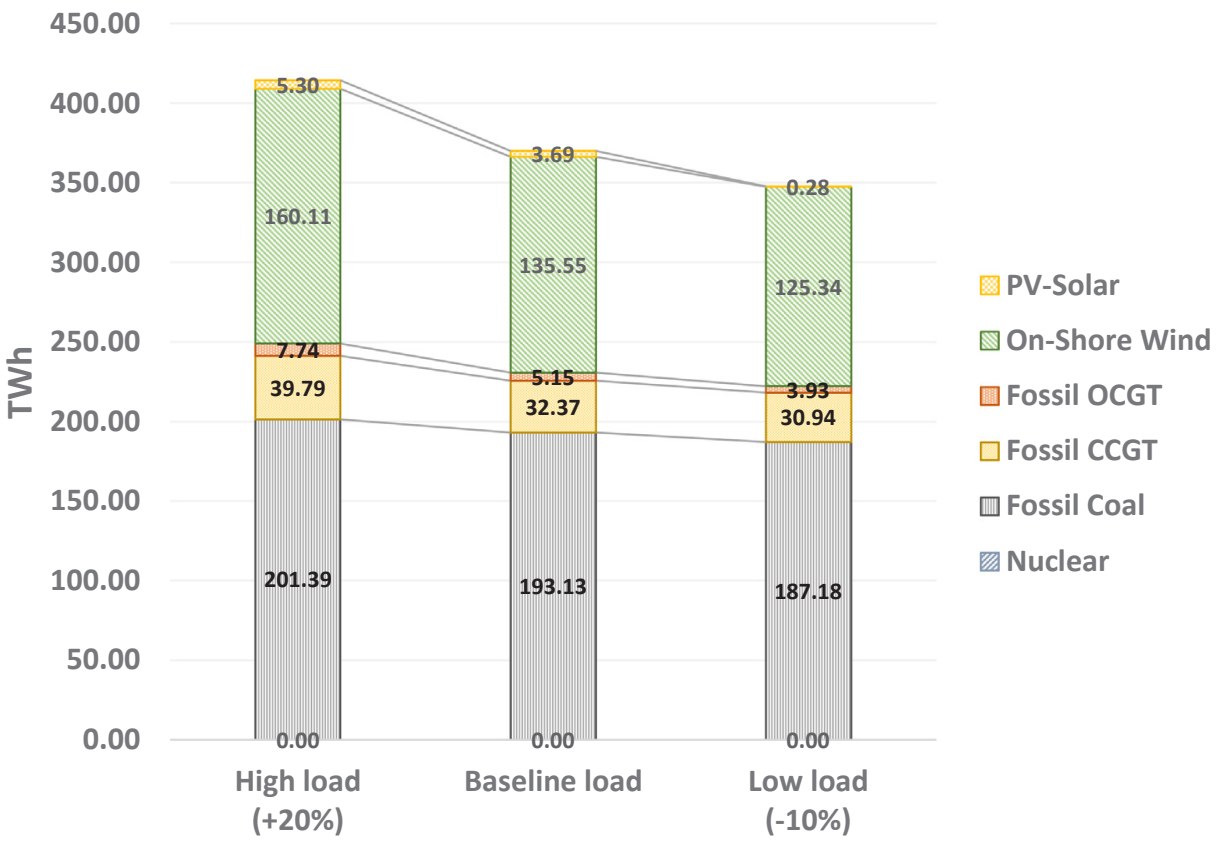

Fig. 5. Total energy production per technology for the plans obtained by the C-GEP model under 35\% RES penetration for the different load growth scenarios. 
Table 5

Results of different performance measures and operational flexibility metrics for the plans obtained through the C-GEP models under 35\% RES penetration for the different load growth scenarios.

(a) Generic performance measures results

\begin{tabular}{lccc} 
& High load (+20\%) & Baseline load & Low load (-10\%) \\
\hline Total RES Installed Capacity [GW] & 94.24 & 78.32 & 69.6 \\
Total Installed Capacity [GW] & 188.52 & 156.83 & 142.15 \\
\hline Load Not Served [\% of total load] & $0.02 \%$ & $0.02 \%$ & $0.02 \%$ \\
RES shedding [\% of total RES] & $3.43 \%$ & $2.48 \%$ & $2.25 \%$ \\
Carbon Emission [Mtons] & 2818.70 & 2648.64 & 2555.18 \\
Total Cost [Bn€] & 439.67 & 383.58 & $357.42 \%$ \\
\hline
\end{tabular}

(b) Operational flexibility metrics results

\begin{tabular}{ccccc} 
& & High load (+20\%) & Baseline load & Low load (-10\%) \\
\hline IRRE+ [\% of upwards ramps] & $1.16 \%$ & $1.12 \%$ & $1.05 \%$ \\
\hline \multirow{2}{*}{ EFS+ [MW] } & EFS+ [95\%] & 51.10 & 38.76 & 32.30 \\
& EFS+ [99\%] & 254.70 & 193.24 & 161.10 \\
\hline IRRE- [\% of downwards ramps] & $0.08 \%$ & $0.04 \%$ & $0.04 \%$ \\
\hline \multirow{2}{*}{ EFS- [MW] } & EFS- [95\%] & 0.00 & 0.00 & 0.00 \\
\hline
\end{tabular}

Report", RTE [30] presented future load projections for France and Europe, with high and low growth scenarios of roughly $+2 \%$ and $-1 \%$, respectively, accounting for all different sectors. For the purpose of sensitivity analysis, for the high an low load scenarios, we have amplified these values considering a $+20 \%$ and a $-10 \%$ load growth starting of the fifth year of the planning horizon. The negative scenario being a proxy for stringent energy efficiency driven policies. For clarity, only the results of the C-GEP model are reported for the median 35\% RES penetration level and for the no emission limit policy. It should also be noted that no changes are assumed for the hourly load patterns compared to the previous cases considered.

The total power generation per technology and per load evolution scenario is illustrated in Fig. 5. The Figure shows that, overall, there are no changes in the capacity mixes obtained, but that only the total capacities and production quantities vary per technology. Naturally, the installed capacities and power generation decrease as the total system load decreases. The reduction is mostly in the RES technologies amounting to more than $50 \%$ of the total generation decrease across scenarios. This can be explained through two effects: lower load means that less RES is required to satisfy the $35 \%$ penetration requirement, and it is more cost efficient (subject to the given assumptions) to reduce the RES levels than to answer to the increased net load variability by cycling thermal units.

Regarding how the load evolution trend affects the obtained plans, Table 5 a summarizes the results of some of the performance measures previously considered. Overall, a gradual decreasing trend can be observed in all measurements with respect to the decreasing load scenario. This is also true for the operational flexibility results reported in Table 5b. While the absolute difference in those results would be important to consider for actual system planning, the linear gradual trend found suggests that the overall load evolution exhibits a less significant effect on the operational flexibility of the plans than the variations in the inter-temporal load patterns.

\section{Conclusions}

In this work, an integrated framework for the quantitative assessment of operational flexibility in power systems planning has been presented and a realistic size case study has been investigated under several scenarios of renewable energy sources penetration levels and carbon reduction targets. Moreover, an investigation of the effect of varying the fuel costs and load growth has been conducted to comprehensively identify the most significant parameters that can affect the system operational flexibility.

The application of the framework to the case studies has shown its ability to provide transparent and objective results for obtaining and assessing different expansion plans across a wide range of policy requirements. The study has also allowed to highlight the importance of integrating short-term technical constraints and chronological load patterns, within long-term planning models and especially under significant renewable energy penetration levels.

Through the analysis of the results of the case studies considered, the following general conclusions can be drawn:

- The results confirm those presented in other works. Most notably, that neglecting short-term constraints within long-term planning leads to an underestimation of the investment required in peaking fossil units, unrealistic production schedules with values of load not served reaching unacceptable levels of up to $3 \%$, and an underestimation of carbon emissions of up to $60 \%$.

- Insights were gained by employing quantitative flexibility metrics for the assessment, most notably that expansion plans obtained through the integrated model are robust to the different renewable energy penetration and emission scenario realizations, in terms of flexibility shortage, i.e., they maintain a constant low shortage level regardless of the different requirements imposed. For the case studies considered, these levels were shown not to exceed expected shortage levels of $2 \%$ in frequency and several MWs in magnitude.

- On the other hand, flexibility-neglecting generation expansion planning models have shown a linear and significant trend of flexibility shortage under increasing renewable energy penetration requirements (up to $50 \%$ in frequency and several GWs in magnitude), enough to offset any computational advantage they have when such requirements are binding.

- The complementarity of the two metrics considered, with regards to the frequency of flexibility shortage and its magnitude, is highlighted. It is shown that the sensitivity of one with respect to the different scenarios can be more significant than the other, which is important to consider for real applications.

- Moreover, the results emphasize the importance of the use of 
suitable quantitative metrics for operational flexibility assessment, as opposed to relying on other generic indicators, such as the generation mix or system costs, which are not capable of reflecting the true flexibility levels of the obtained plans.

Finally, a limitation of the presented work lies in the mixed integer linear optimization models considered. Indeed, the economic planning parameters and the technical behavior of energy generation are affected by nonlinear conditions. For instance, production costs and ramping rates are nonlinear functions of the variations in partial-load levels, while start-up costs and times are nonlinear functions of shut-down duration. These conditions become particularly relevant when shortterm capabilities and operational flexibility are considered in the model. In this regard, future work will be devoted to the extension of the optimization model for accounting of nonlinearities in the system. Moreover, the model can be further extended by considering muti-regional planning, network representation and the uncertainties in the load and renewable energy generation.

\section{Appendix A. Models formulation}

\section{A.1. Long-term GEP model with no short-term constraints}

The model is formulated as a mixed integer linear program (MILP), with the main characteristics that no hourly chronological order is considered and demand is represented as load blocks derived from a load-duration curve with durations (Dury,s $)$ and levels $\left(L_{y, s}\right)$.

\section{A.1.1. GEP objective function}

The objective is the minimization of the total discounted costs over the planning horizon. Eq. (A.1) represents the total investment costs in new units, Eq. (A.2) represents the total production costs calculated on the basis of the yearly load-duration curves, and Eq. (A.3) represents the fixed operation and maintenance (O\&M) costs:

$$
\begin{aligned}
& \min _{\text {cost }} \sum_{y \in Y}(1+D R)^{-y} \cdot \sum_{i \in I} C_{i}^{i n v} \cdot P_{i}^{\text {max }} \cdot q_{i, y} \\
& +\sum_{y \in Y}(1+D R)^{-y} \cdot \sum_{s \in S} \operatorname{Dur}_{y, s} \cdot\left[\sum_{i \in I}\left(C_{i, y}^{\text {marginal }} \cdot p_{i, y, s}\right)+C^{l n s} \cdot \ln s_{y, s}\right] \\
& +\sum_{y \in Y}(1+D R)^{-y} \cdot \sum_{i \in I} C_{i, y}^{\text {fom }} \cdot P_{i}^{\max } \cdot \sum_{l=1}^{y} q_{i, l}
\end{aligned}
$$

\section{A.1.2. GEP constraints}

1. Units commissioning and construction time:

$$
x_{i, y}=\sum_{l=1}^{y} q_{i, l-T_{i}^{\text {const }}+1} \quad \forall i \in I^{\text {new }}, y \in Y
$$

2. Annual budget constraint:

$$
\sum_{y \in Y} q_{i, y} \leqslant I_{i}^{\max } \quad \forall i \in I^{\text {new }}
$$

3. Lifetime of new units:

$$
\sum_{\tau=y-T_{i}^{l i f e}+1}^{y} q_{i, \tau}=x_{i, y} \quad \forall i \in I^{\text {new }}, y \in Y
$$

4. Supply-demand balance constraint:

$$
\operatorname{Dur}_{y, s}\left(\sum_{i \in I} p_{i, y, s}+\ln s_{y, s}\right)=\operatorname{Dur}_{y, s} \cdot L_{y, s} \quad \forall s \in S, y \in Y
$$

5. Maximum generation output levels:

$$
\sum_{s \in S} p_{i, y, s} \leqslant\left(1-E F O R_{i}\right) \cdot P_{i}^{\max } \cdot x_{i, y} \quad \forall i \in I^{\text {thermal }}, y \in Y
$$

6. Adequacy reserve margin:

$$
\sum_{i \in I}\left(P_{i}^{\max } \cdot x_{i, y}\right) \geqslant\left(1+r^{\min }\right) \cdot \max _{s}\left(L_{y, s}\right), \quad \forall y \in Y
$$

7. Minimum annual renewable penetration: 


$$
\sum_{i \in I^{r e s}} \sum_{s \in S}\left(\text { Dur }_{y, s} \cdot p_{i, y, s}\right) \geqslant \operatorname{Pen}_{y}^{\text {level }} \cdot \sum_{s \in S}\left(\operatorname{Dur}_{y, s} \cdot L_{y, s}\right), \quad \forall y \in\left[Y^{\text {res }}, Y^{\text {end }}\right]
$$

8. Renewable energy production: RES production is typically represented through an hourly availability or hourly capacity factor (CF). Since in the S-GEP problem no chronological hourly representation is considered, an approximation method is used to obtain what we refer to as the RES capacity factor duration curve (RES-CFDC), described in details in Appendix B:

$\operatorname{Dur}_{y, s} \cdot p_{i, y, s} \leqslant x_{i, y} \cdot P_{i}^{\max } \cdot \operatorname{Dur}_{y, s} \cdot C F_{i, y, s} \quad \forall i \in I^{r e s}, s \in S, y \in Y$

9. Allowable emission:

$$
\sum_{i \in I}\left(E_{i} \cdot \sum_{s \in S}\left(\text { Dur }_{y, s} \cdot p_{i, y, s}\right)\right) \leqslant E_{y}^{\max }, \quad \forall y \in Y
$$

\section{A.2. Short-term operational model with no design variables (UC)}

For the UC model, all the commissioning decisions are considered to have been taken beforehand (from the GEP model) and the model seeks only to find the optimal short-term system operation.

\section{A.2.1. UC objective function}

The objective is the minimization of the total discounted operating costs of the systems, including variable production cost, start-up cost and LNS cost:

$\min _{\text {cost }} \sum_{y \in Y}(1+D R)^{-y} \cdot \sum_{j \in J}\left[\sum_{i \in I}\left(C_{i, y}^{\text {marginal }} \cdot p_{i, y, j}+C_{i}^{s} \cdot z_{i, y, j}\right)+C^{\left.\operatorname{lns} \cdot \ln s_{y, j}\right]}\right.$

\section{A.2.2. UC constraints}

1. Only units commissioned can be operated: this constraint represents the link between the long-term investment and the operating decisions:

$u_{i, y, j} \leqslant x_{i, y} \quad \forall i \in I^{\text {thermal }}, j \in J, y \in Y$

2. Supply-demand balance constraint:

$$
\sum_{i \in I} p_{i, y, j}+\ln s_{y, j}=L_{y, j} \quad \forall j \in J, y \in Y
$$

3. Unit-commitment constraint:

$$
u_{i, y, j}-u_{i, y, j-1}=z_{i, y, j}-v_{i, y, j} \quad \forall i \in I^{t h}, j \in J /\{1\}, y \in Y
$$

4. Minimum up-time (A.17) and down-time (A.18) of generation units:

$$
\begin{aligned}
& u_{i, y, j} \geqslant \sum_{\tau \geqslant j-M_{i}^{u}}^{j} z_{i, y, \tau} \quad \forall i \in I^{\text {th }}, j \in J, y \in Y \\
& x_{i, y}-u_{i, y, j} \geqslant \sum_{\tau \geqslant j-M_{i}^{d}}^{j} v_{i, y, \tau} \quad \forall i \in I^{t h}, j \in J, y \in Y
\end{aligned}
$$

5. Upwards (A.19) and downwards (A.20) ramping capabilities of generation units:

$$
\begin{aligned}
& p_{i, y, j}-p_{i, y, j-1} \leqslant u_{i, y, j-1} \cdot R_{i}^{\text {Umax }}+z_{i, y, j} \cdot P_{i}^{\text {start }} \quad \forall i \in I^{\text {th }}, j \in J /\{1\}, y \in Y \\
& p_{i, y, j-1}-p_{i, y, j} \leqslant u_{i, y, j-1} \cdot R_{i}^{\text {Dmax }} \quad \forall i \in I^{\text {th }}, j \in J /\{1\}, y \in Y
\end{aligned}
$$

6. Maximum (A.21) and minimum (A.22) output levels of generation units:

$$
p_{i, y, j}+p r_{i, y, j}+s r_{i, y, j}^{u p} \leqslant\left(1-E F O R_{i}\right) \cdot P_{i}^{\max } \cdot u_{i, y, j} \quad \forall i \in I^{\text {th }}, j \in J, y \in Y
$$




$$
p_{i, y, j} \geqslant u_{i, y, j} \cdot P_{i}^{\min }+s r_{i, y, j}^{d n} \quad \forall i \in I^{t h}, j \in J, y \in Y
$$

7. Operating reserves: three types of operating reserves are considered, according to a defined percentage of hourly load and of renewable generation: those are primary reserve (A.23), secondary upwards reserve (A.24), and secondary downwards reserve (A.25):

$$
\begin{aligned}
& \sum_{i \in I^{t h}} p r_{i, y, j} \geqslant \operatorname{Prr} \cdot L_{y, j} \quad \forall j \in J, y \in Y \\
& \sum_{i \in I^{t h}} s r_{i, y, j}^{u p} \geqslant S r r^{u p} \cdot L_{y, j}+\sum_{i \in I^{r e s}}\left(a^{r e s} \cdot p_{i, y, j}\right) \quad \forall j \in J, y \in Y \\
& \sum_{i \in I^{t h}} s r_{i, y, j}^{d n} \geqslant S r r^{d n} \cdot L_{y, j}+\sum_{i \in I^{r e s}}\left(a^{r e s} \cdot p_{i, y, j}\right) \quad \forall j \in J, y \in Y
\end{aligned}
$$

8. Renewable energy production:

$$
p_{i, y, j} \leqslant x_{i, y} \cdot P_{i}^{\max } \cdot C F_{i, y, j} \quad \forall i \in I^{r e s}, j \in J, y \in Y
$$

\section{Appendix B. RES-CF approximation for the long-term GEP model}

One way to approximate the RES-CF is to re-order the chronological CF values in descending order and divide them into CF-blocks, each having a level and duration. A fundamental problem with this approach is that it presumes that the highest RES-CF is concurrent with the highest load level and, analogously, the lowest RES-CF is concurrent with the lowest load level. This imposes a significant and unrealistic bias in the results. We, thus, propose to approximate the RES capacity factor duration curve (RES-CFDC) in a way that maintains the real hourly correlation between the load and the RES availability, when both chronological time-series are available.

This can be best illustrated by means of an example: consider a 6 weeks representation of hourly load and solar CF time series, such as that shown in Fig. B.6. This can be a forecasted time-series or historically monitored data. Each hourly load level corresponds to a specific solar CF for the same hour. When the load is re-ordered in descending order into a LDC, the solar-CF is re-ordered by maintaining each CF respective value relative to its original hourly load level. When the LDC is, then, approximated by a step-function to obtain average load levels and durations, the same duration blocks are used to segment and find corresponding average values for the solar CF time-series.
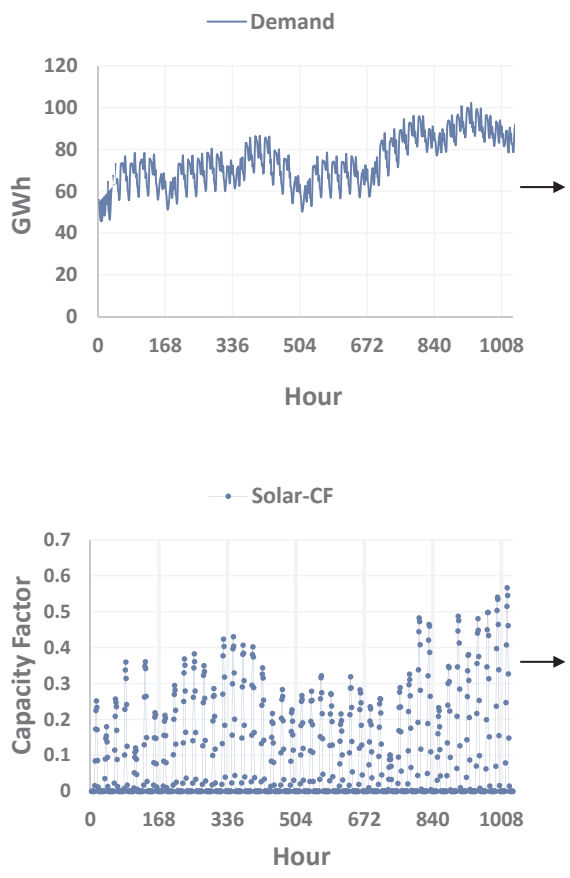

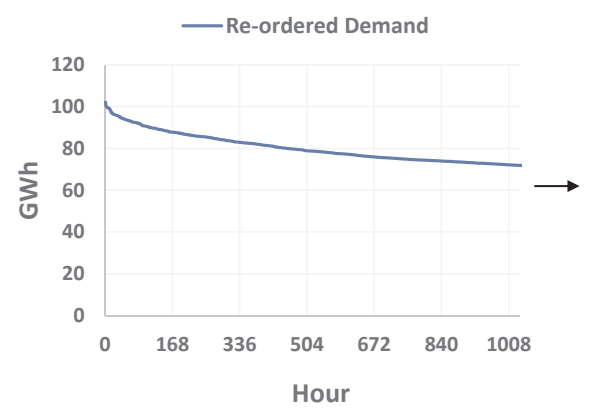

(a) LDC step function approximation
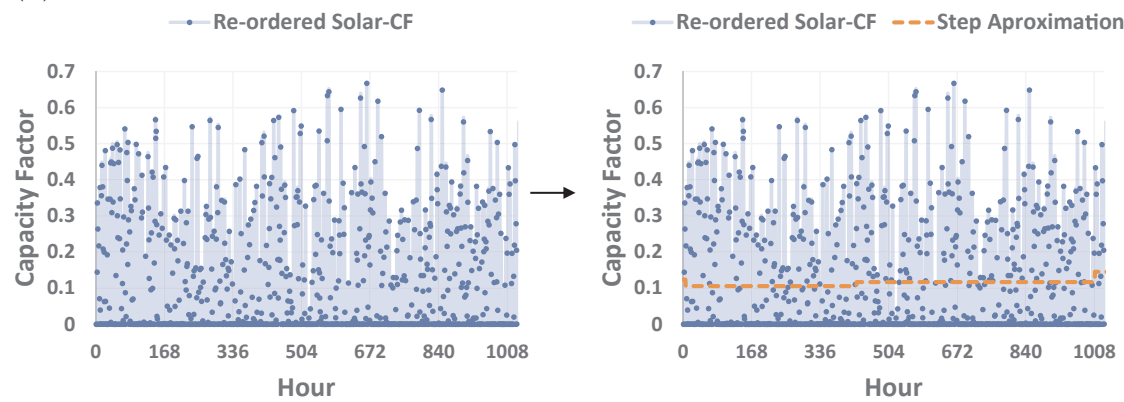

(b) Solar-CF step function approximation

Fig. B.6. 6 weeks representation of load and Solar CF yearly data and their step function approximation. 


\section{Appendix C. Load profiles}

See Fig. C.7.

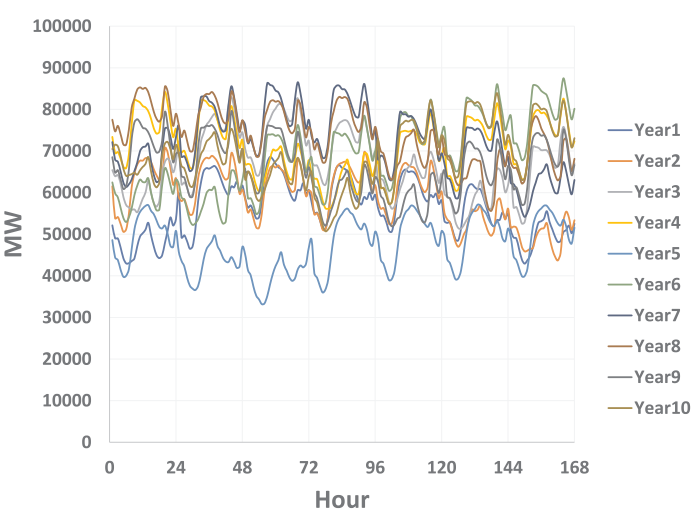

(a) Week 1

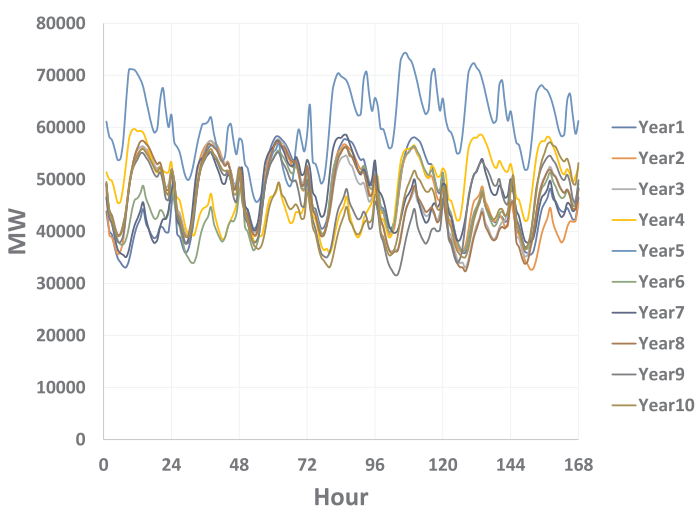

(c) Week 3

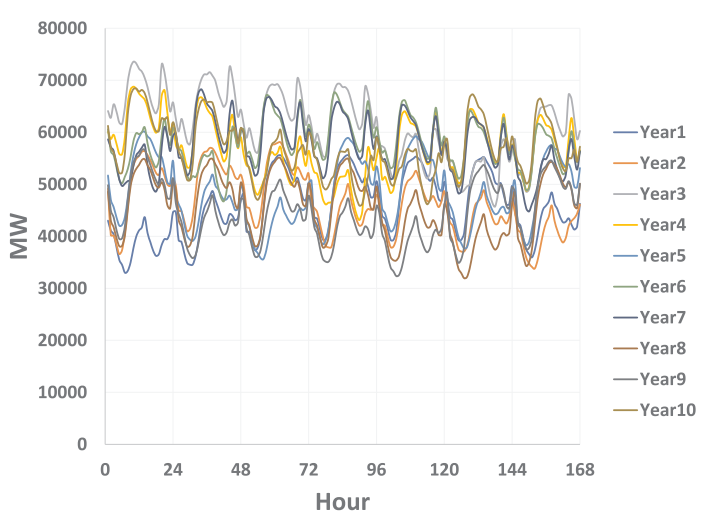

(b) Week 2

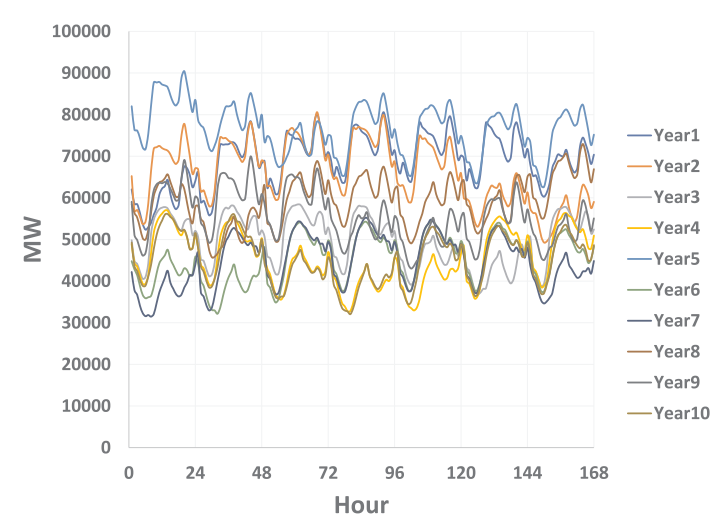

(d) Week 4

Fig. C.7. Weekly load profile samples for each year of the planning horizon considered (illustration of the wide variation in inter-temporal variability considered in the study).

\section{References}

[1] Sadeghi Hadi, Rashidinejad Masoud, Abdollahi Amir. A comprehensive sequential review study through the generation expansion planning. Renew Sustain Energy Rev 2017;67:1369-94.

[2] Oree Vishwamitra, Sayed Hassen Sayed Z, Fleming Peter J. Generation expansion planning optimisation with renewable energy integration: a review. Renew Sustain Energy Rev 2017;69:790-803. ISSN 1364-0321.

[3] Caunhye Aakil M, Cardin Michel-Alexandre. An approach based on robust optimization and decision rules for analyzing real options in engineering systems design. IISE Trans 2017;49(8):753-67.

[4] European Council. Directive 2009/28/ec of the European parliament and of the council of 23 April 2009 on the promotion of the use of energy from renewable sources and amending and subsequently repealing directives 2001/77/ec and 2003/30/ec; 2009 . < http://eur-lex.europa.eu/eli/dir/2009/28/oj > [accessed on 02-01-2017].

[5] European Council. Proposal for a directive of the European parliament and of the council on the promotion of the use of energy from renewable sources (recast) com/2016/0767 final/2 - 2016/0382 (cod); 2016. < http://eur-lex.europa.eu/legal-content/EN/ALL/? uri $=$ CELEX:52016PC0767R(01) > [accessed on 02-01-2017]

[6] Brouwer Anne Sjoerd, van den Broek Machteld, Seebregts Ad, Faaij Andr. Operational flexibility and economics of power plants in future low-carbon power systems. Appl Energy 2015;156:107-28. ISSN 0306-2619.

[7] Kubik ML, Coker PJ, Barlow JF. Increasing thermal plant flexibility in a high renewables power system. Appl Energy 2015;154:102-11. ISSN 0306-2619.

[8] Fulli Gianluca, Masera Marcelo, Covrig Catalin Felix, Profumo Francesco, Bompard Ettore, Huang Tao. The eu electricity security decision-analytic framework: status and perspective developments. Energies 2017;10(4):425.

[9] Lannoye Eamonn, Flynn Damian, O'Malley Mark. Evaluation of power system flexibility. IEEE Trans Power Syst 2012;27(2):922-31.

[10] Oree Vishwamitra, Sayed Hassen Sayed Z. A composite metric for assessing flexibility available in conventional generators of power systems. Appl Energy 2016;177:683-91.

[11] Ulbig Andreas, Andersson Göran. Analyzing operational flexibility of electric power systems. Int J Electr Power Energy Syst 2015;72:155-64.
[12] Zhao Jinye, Zheng Tongxin, Litvinov Eugene. A unified framework for defining and measuring flexibility in power system. IEEE Trans Power Syst 2016;31(1):339-47.

[13] Abujarad Saleh Y, Mustafa MW, Jamian JJ. Recent approaches of unit commitment in the presence of intermittent renewable energy resources: a review. Renew Sustain Energy Rey 2017;70:215-23.

[14] Belderbos Andreas, Delarue Erik. Accounting for flexibility in power system planning with renewables. Int J Electr Power Energy Syst 2015;71:33-41.

[15] Palmintier Bryan S, Webster Mort D. Impact of operational flexibility on electricity generation planning with renewable and carbon targets. IEEE Trans Sustain Energy 2016;7(2):672-84.

[16] Welsch Manuel, Deane Paul, Howells Mark, Gallachóir Brian Ó, Rogan Fionn, Bazilian Morgan, et al. Incorporating flexibility requirements into long-term energy system models - a case study on high levels of renewable electricity penetration in Ireland. Appl Energy 2014;135:600-15.

[17] Wierzbowski Michal, Lyzwa Wojciech, Musial Izabela. Milp model for long-term energy mix planning with consideration of power system reserves. Appl Energy 2016;169:93-111. ISSN 0306-2619.

[18] Koltsaklis Nikolaos E, Georgiadis Michael C. A multi-period, multi-regional generation expansion planning model incorporating unit commitment constraints. Appl Energy 2015;158:310-31.

[19] Guo Zheng, Cheng Rui, Xu Zhaofeng, Liu Pei, Wang Zhe, Li Zheng, et al. A multi-region load dispatch model for the long-term optimum planning of China's electricity sector. Appl Energy 2017;185:556-72. ISSN 0306-2619.

[20] Pereira Sérgio, Ferreira Paula, Vaz AIF. Generation expansion planning with high share of renewables of variable output. Appl Energy 2017;190:1275-88.

[21] Palmintier Bryan S, Webster Mort D. Heterogeneous unit clustering for efficient operational flexibility modeling. IEEE Trans Power Syst 2014;29(3):1089-98.

[22] Maybee John, Randolph Paul, Uri Noel. Optimal step function approximations to utility load duration curves. Eng Optim 1979;4(2):89-93.

[23] De Sisternes Fernando J, Webster Mort D. Optimal selection of sample weeks for approximating the net load in generation planning problems. In: Massachusetts Institute of Technology Engineering Systems Division Working Paper Series, vol. 3; 2013. p. 1-12.

[24] Yamai Yasuhiro, Yoshiba Toshinao. Value-at-risk versus expected shortfall: a practical perspective. J Bank Finan 2005;29(4):997-1015. 
[25] RTE-France; 2017. Available at:. < http://clients.rte-france.com > [accessed on 20-122016].

[26] IEA/NEA. Projected costs of generating electricity 2015 edition. OECD; 2015

[27] Cany Camille, Mansilla Christine, Costa Pascal da, Mathonnire Gilles, Duquesnoy Thierry, Baschwitz Anne. Nuclear and intermittent renewables: two compatible supply options? The case of the French power mix. Energy Policy 2016;95:135-46.

[28] Hart William E, Laird Carl, Watson Jean-Paul, Woodruff David L. Pyomo-optimization modeling in python vol. 67. Springer Science \& Business Media; 2012.

[29] Hart William E, Watson Jean-Paul, Woodruff David L. Pyomo: modeling and solving mathematical programs in python. Math Programm Comput 2011:3(3):219-60.

[30] Réseau de transport d'eélectriciteé RTE. Generation adequacy report on the electricity supply-demand balance in france - 2016 edition; 2016. < http://www.rte-france.com/ en/article/forecast-assessment-electricity-supply-demand-balance $>$ [accessed on 02-12 2017]. 